\title{
ARMCX Family Gene Expression Analysis and Potential Prognostic Biomarkers for Prediction of Clinical Outcome in Patients with Gastric Carcinoma
}

\author{
TingAn Wang, ${ }^{1}$ HuaGe Zhong, ${ }^{1}$ YuZhou Qin, ${ }^{1}$ WeiYuan Wei, ${ }^{1}$ Zhao Li, ${ }^{1}$ MingWei Huang $\mathbb{D}^{1}$ \\ and XiaoLing Luo $\mathbb{D}^{2}$ \\ ${ }^{1}$ Department of Gastrointestinal Surgery, Guangxi Medical University Cancer Hospital, Guangxi Clinical Research Center for \\ Colorectal Cancer, Nanning 530021, Guangxi Zhuang Autonomous Region, China \\ ${ }^{2}$ Guangxi Medical University Cancer Hospital, Nanning 530021, Guangxi Zhuang Autonomous Region, China
}

Correspondence should be addressed to MingWei Huang; mingweihuang1@163.com and XiaoLing Luo; luoxiaoling67@126.com

TingAn Wang and HuaGe Zhong contributed equally to this work.

Received 12 February 2020; Accepted 20 May 2020; Published 30 June 2020

Academic Editor: Ji-Fu Wei

Copyright (c) 2020 TingAn Wang et al. This is an open access article distributed under the Creative Commons Attribution License, which permits unrestricted use, distribution, and reproduction in any medium, provided the original work is properly cited.

\begin{abstract}
Armadillo gene subfamily members (ARMCX1-6) are well-known to regulate protein-protein interaction involved in nuclear transport, cellular connection, and transcription activation. Moreover, ARMCX signals on cell pathways also implicated in carcinogenesis and tumor progression. However, little is known about the associations of the ARMCX subfamily members with gastric carcinoma. This study investigated the prognostic value of ARMCX subfamily mRNA expression levels with the prognosis of gastric carcinoma (GC). We retrieved the data of a total of 351 GC patients from TCGA database. Survival and gene set enrichment analyses were employed to explore the predictive value and underlying mechanism of ARMCX genes in GC. The multivariate survival analysis revealed that individually low expressions of ARMCX1 (adjusted $P=0.006, \mathrm{HR}=0.620$, $\mathrm{CI}=0.440-0.874$ ) and ARMCX2 (adjusted $P=0.005, \mathrm{HR}=0.610,95 \% \mathrm{CI}=0.432-0.861$ ) were related to preferable overall survival (OS). The joint-effects analysis shown that combinations of low level expression of ARMCX1 and ARMCX2 were correlated with favorable OS (adjusted $\mathrm{P}=0.003, \mathrm{HR}=0.563,95 \% \mathrm{CI}=0.384-0.825$ ). ARMCX1 and ARMCX2 were implicated in WNT and NF-kappaB pathways, and biological processes including cell cycle, apoptosis, RNA modification, DNA replication, and damage response. Our results suggest that mRNA expression levels of ARMCX subfamily are potential prognostic markers of GC.
\end{abstract}

\section{Introduction}

Gastric carcinoma, one common type of malignant tumors, is the fifth highest incidence and the second highest mortality after lung cancer worldwide [1]. Each year, more than 300,000 newly diagnosed cases and about 260,000 people die in China. The poor prognosis is due to a high incidence of advanced disease, high recurrence rate, high metastasis, and abnormal gene expression. In addition, despite great advances in the surgery and chemotherapy technology, the death rate remains high [2]. Therefore, new strategies to improve diagnosis and prognosis of gastric cancer are shortly needed.
The armadillo genes are clustered on the $\mathrm{X}$ chromosome, also known as X-linked (ARMCX or ALEX). In 1989, it was first discovered in the segment polarity gene armadillo in Drosophila $[3,4]$. Since then, more and more related proteins have been identified and classified as armadillo repeat family. The common feature of these proteins is an amino acid sequence (arm repeats) approximately 42 residues, identified as 6-13 repeat units in all members of the family $[5,6]$, and each repeat domain consists of three helices, designated as $\mathrm{H} 1, \mathrm{H} 2$, and $\mathrm{H} 3$ [7-9].

The armadillo domain protein has the functions of cell contact and cytoskeletal-related protein and signal 


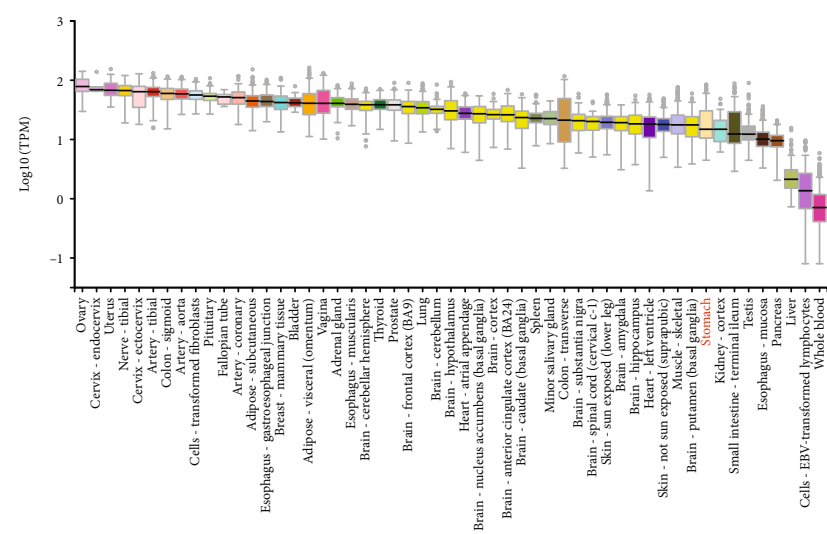

(a)

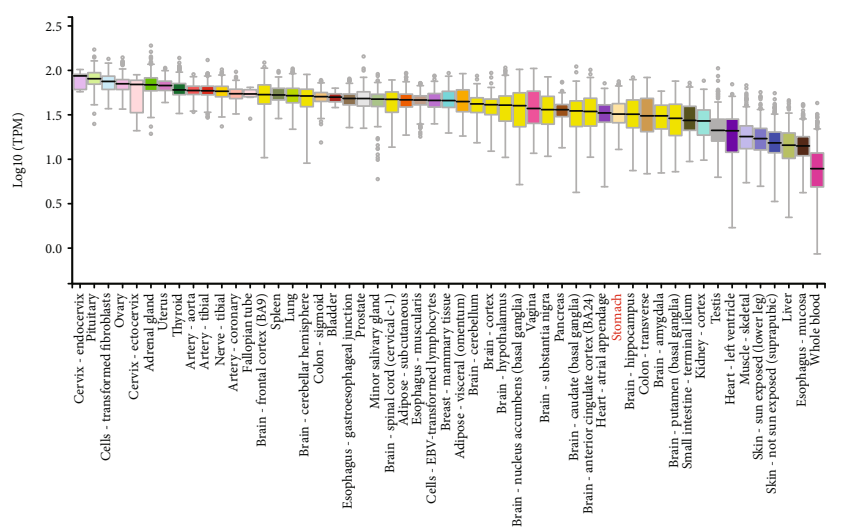

(c)

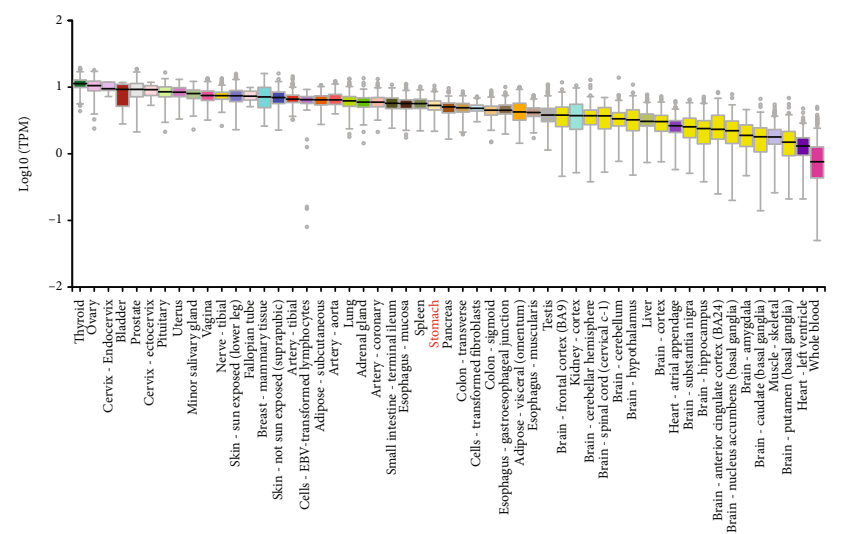

(e)

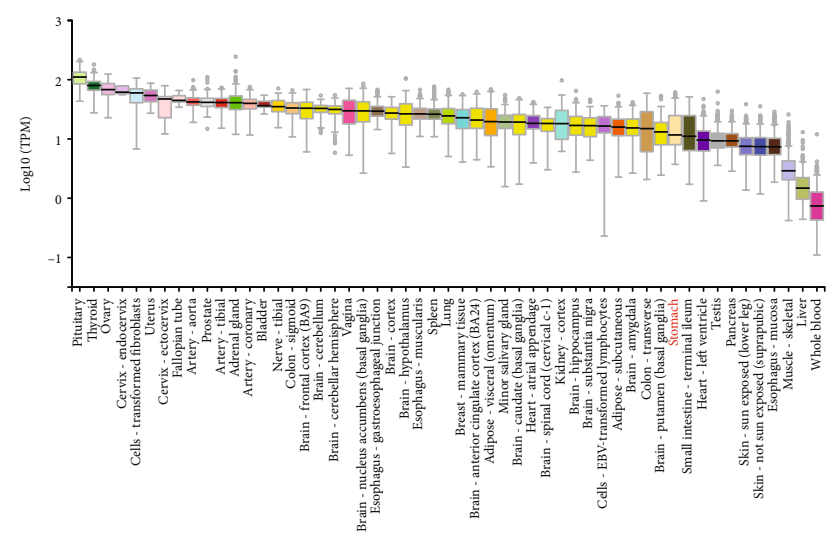

(b)

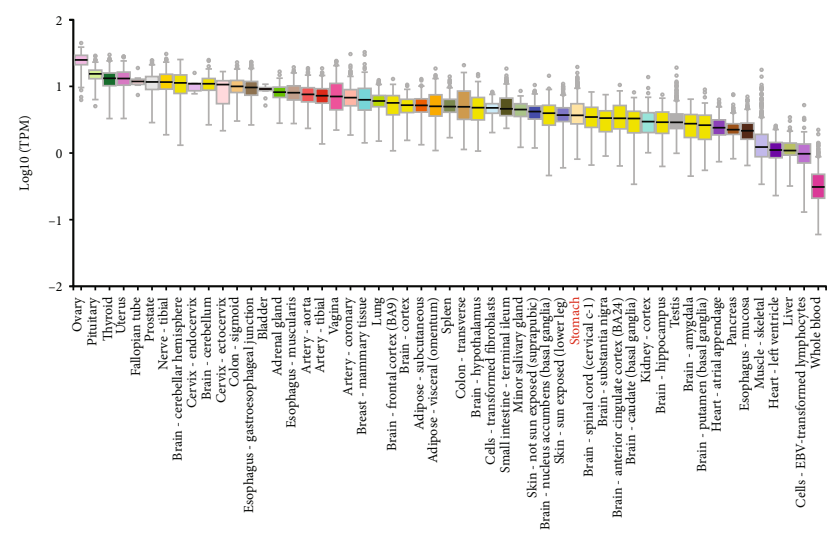

(d)

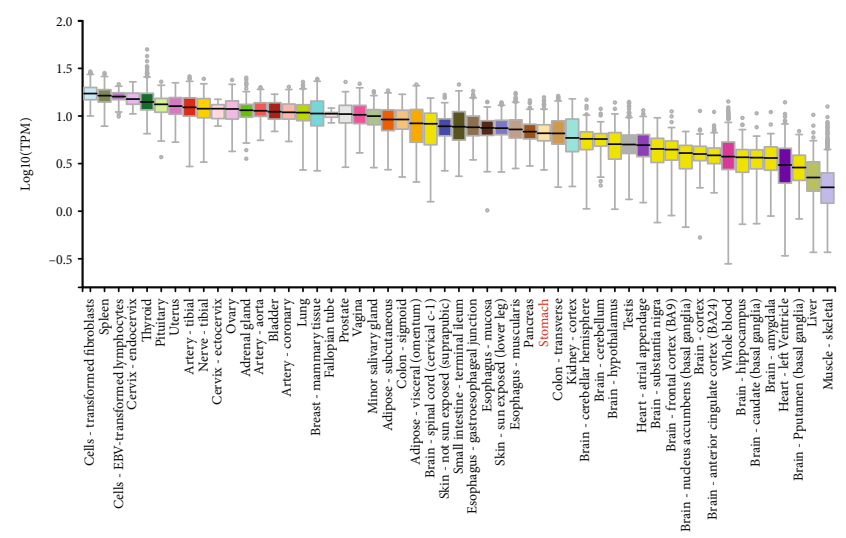

(f)

FIGURE 1: Matrix graphs of the relative expression levels of ARMCX genes in multiple normal tissues were determined with the GTEx Portal. ARMCX5 was expressed at a medium level (e), whereas the other ARMCX genes (ARMCX1, ARMCX2, ARMCX3, ARMCX4, and ARMCX6) were expressed at low levels (a-d, f).

transmission by producing and transmitting signals that affect gene expression [5,9]. Studies have revealed that armadillo repeat proteins regulate protein interactions through multiple binding domains such as nuclear transport, transcriptional activation, and cell connectivity [10]. For example, bioinformatics analysis shows that ARMCX1, ARMCX2, and ARMCX3 are encoded by an single exon, containing some ARM repeat domains, a DUF634 (domain 634 function unknown) and an N-terminal transmembrane domain [11-13].
Recent studies have shown a strong implication of different members of the Armcx1-6/Armc10 family in human tumorigenesis [14-16]. For instance, some members of the Armcx cluster can be regulated through the WNT signaling pathway by interacting with transcription factors of the E-cadherin and $\mathrm{T}$ cytokine/lymphoid enhancement factor (TCF/LEF) families [17, 18], which is also implicated in carcinogenesis and tumor progression [19-21].

Although the ARMCX family plays an important role in many biological processes including cell adhesion, 


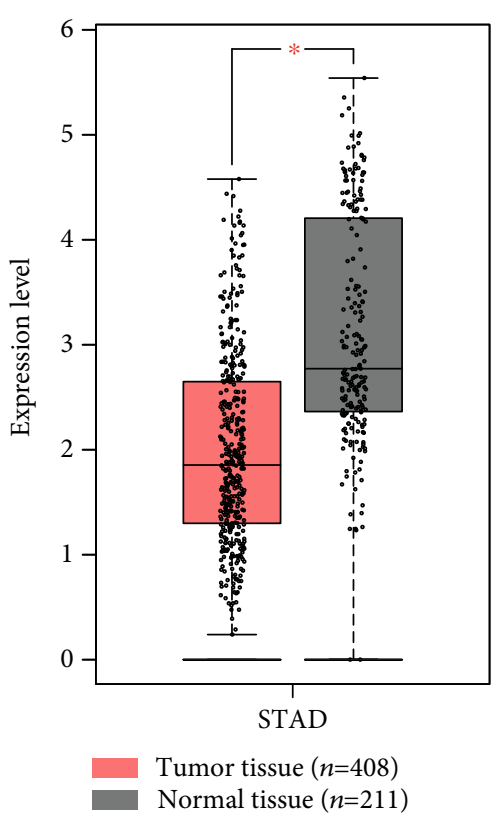

(a)

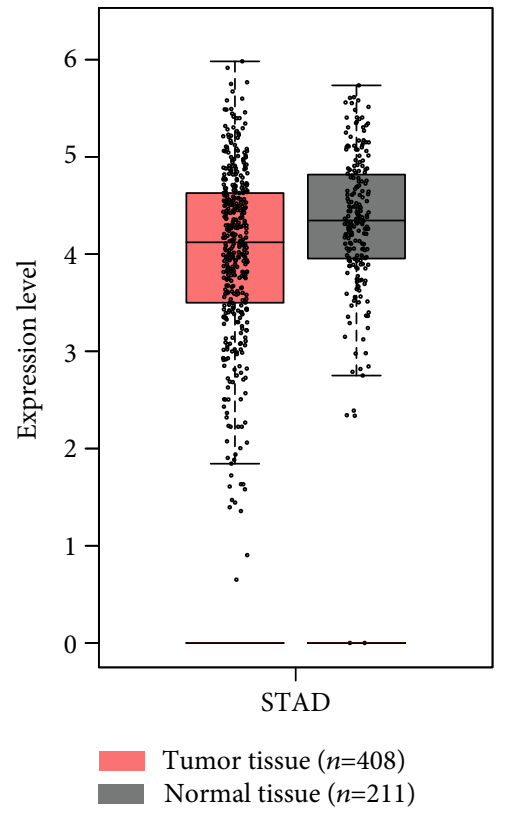

(c)

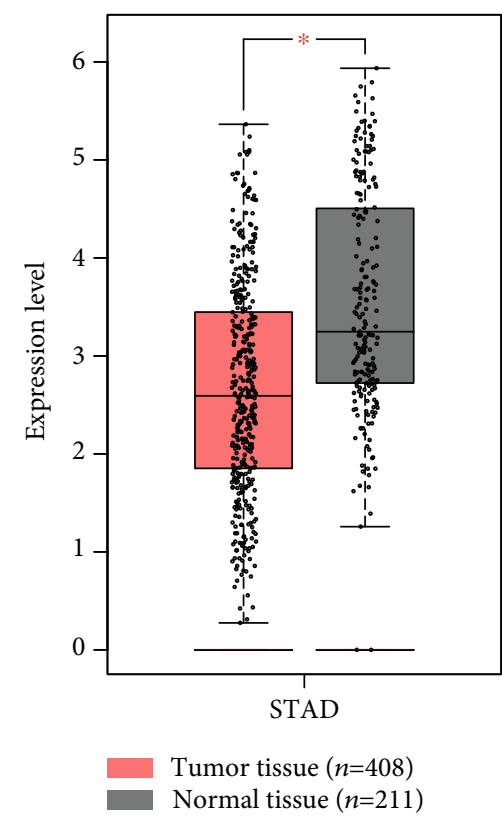

(b)

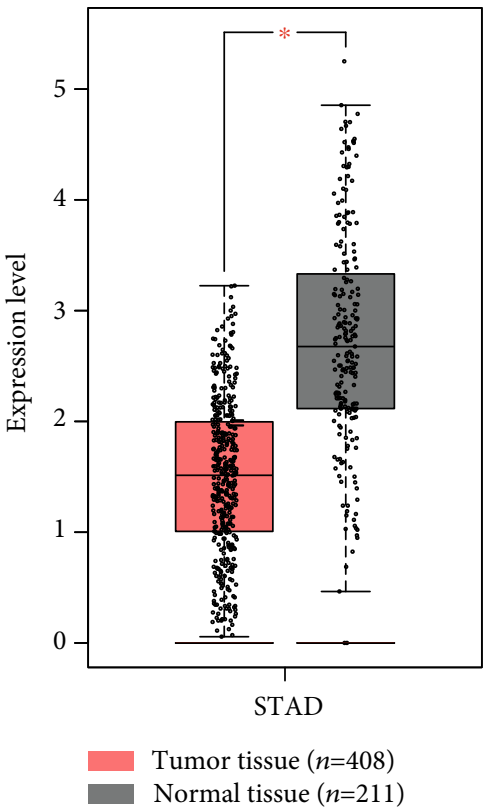

(d)

Figure 2: Continued. 


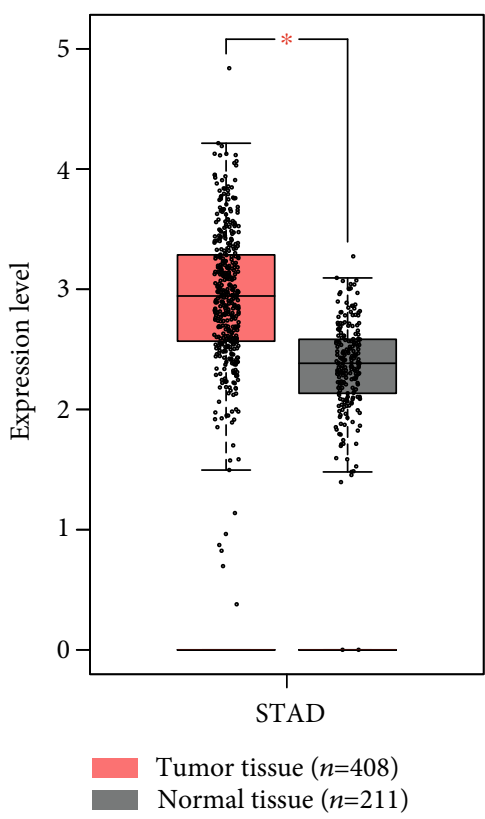

(e)

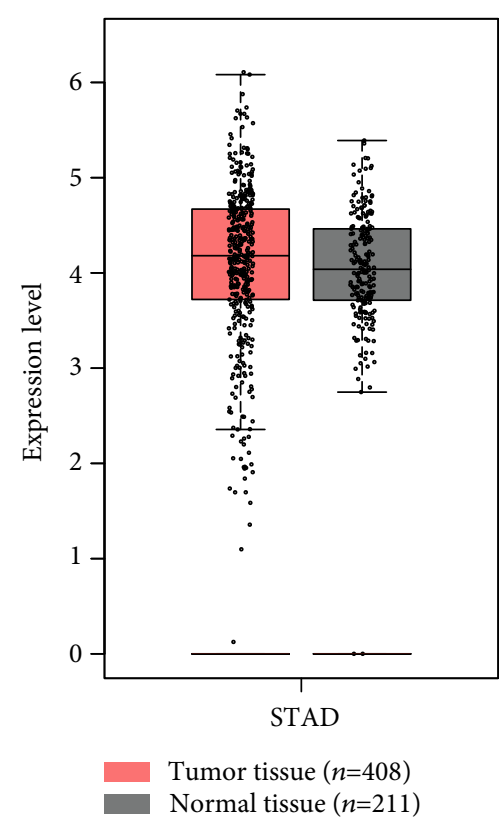

(f)

FIGURE 2: Boxplots of ARMCX family gene levels in primary gastric tumors and adjacent tissues from databases. ARMCX1, ARMCX2, and ARMCX4 were lowly expressed in primary gastric tumors (a, b, d). ARMCX5 was less expressed in normal gastric tissues (e). The expression levels of ARMCX3 and ARMCX6 have no significant difference between gastric tumors and normal gastric tissues $(c, f) .{ }^{*} P<0.05$.

tumorigenesis, and embryogenesis [22]. However, the relationship between ARMCX genes and gastric cancer is poorly understood. Therefore, in this study, we determined the associations between expression levels of ARMCX genes and clinical outcomes of GC prognosis, with the aim of providing insightful information regarding ARMCX genes as a novel prognostic biomarker for GC patients.

\section{Material and Methods}

2.1. Data Source and Patient Information. First, we identified the genes differentially expressed between normal gastric tissue and primary tumors of the ARMCX family using an online database (http://merav.wi.mit.edu/; accessed Sept 25, 2019). Then, we obtained mRNA expression levels of ARMCX1, ARMCX2, ARMCX3, ARMCX4, ARMCX5, and ARMCX6 by using The Cancer Genome Atlas (TCGA, http://tcga-data.nci.nih.gov/tcga) and OncoLnc website (http://www.oncolnc.org/; accessed Sept 25, 2019) [23].

We downloaded the clinical information of 415 gastric cancer patients from UCSC Xena (http://xena.ucsc.edu/, accessed Sept 25, 2019), including age, gender, tumor stage, survival time, and survival status. Next, a total of 351 cases were included for follow-up analysis after excluding the cases with missing medical data and 0 -day survival time.

2.2. Characteristics of Gene Expression Levels. The highexpression and low-expression groups of ARMCX genes were distinguished according to the median of each gene. The relative expression levels of ARMCX genes in multiple normal tissues were determined with the GenotypeTissue Expression Portal (http://www.gtexportal.org/home/, accessed Sept 25, 2019) [24].The analysis of ARMCX mRNA expression between primary gastric cancer tissue and adjacent normal tissue was done by Gene Expression Profiling Interactive Analysis (GEPIA, http:/gepia.cancer-pku.cn/, accessed Sept 25, 2019) [25].

2.3. Bioinformatics Characteristic of ARMCX Genes. Gene function enrichment analysis of ARMCX genes was performed to disclose the biological processes and signal pathways using the Database for Annotation and Enrichment KOBAS 3.0 (http://kobas.cbi.pku.edu.cn/index.php). The analysis included biological processes and molecular function, but no results for the ARMCX family were obtained. GeneMANIA was employed to reveal the genegene and protein-protein interactions of ARMCX family (http://www.genemania.org/, accessed Sept 26, 2019) [26, 27]. Additionally, the relationship among ARMCX1, ARMCX2, ARMCX3, ARMCX4, ARMCX5, and ARMCX6 was evaluated using Pearson's correlation coefficient. Results with a $P$ value $<0.001$ were considered to be statistically significant.

2.4. Survival Analysis. According to the database, the $351 \mathrm{GC}$ patients were, respectively, divided into low- and highexpression groups for survival analysis. Overall survival (OS) and median survival time (MST) were used to assess the prognosis of patients with gastric cancer, to evaluate the correlation of ARMCX member mRNAs with patient survival by Kaplan-Meier estimator with a log-rank test. The relative risk of survival in gastric cancer patients was assessed by calculating the hazard ratio (HR) and $95 \%$ confidence interval (CI). 


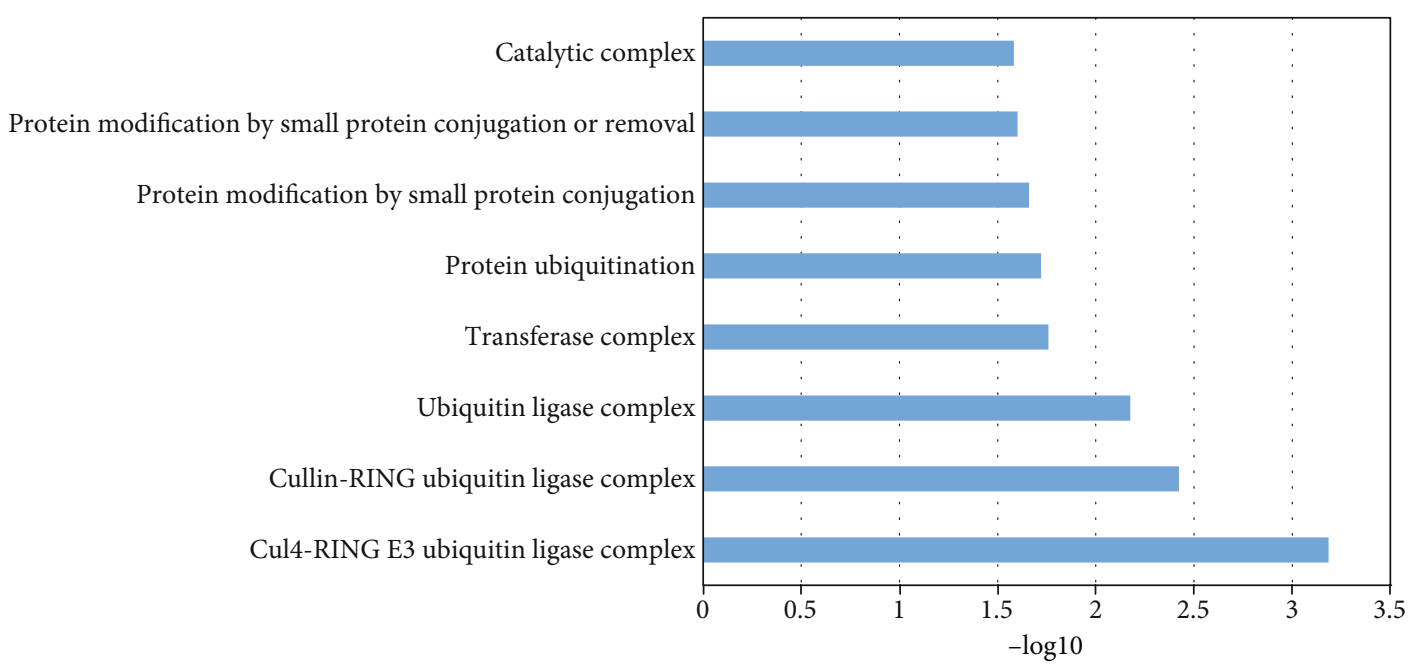

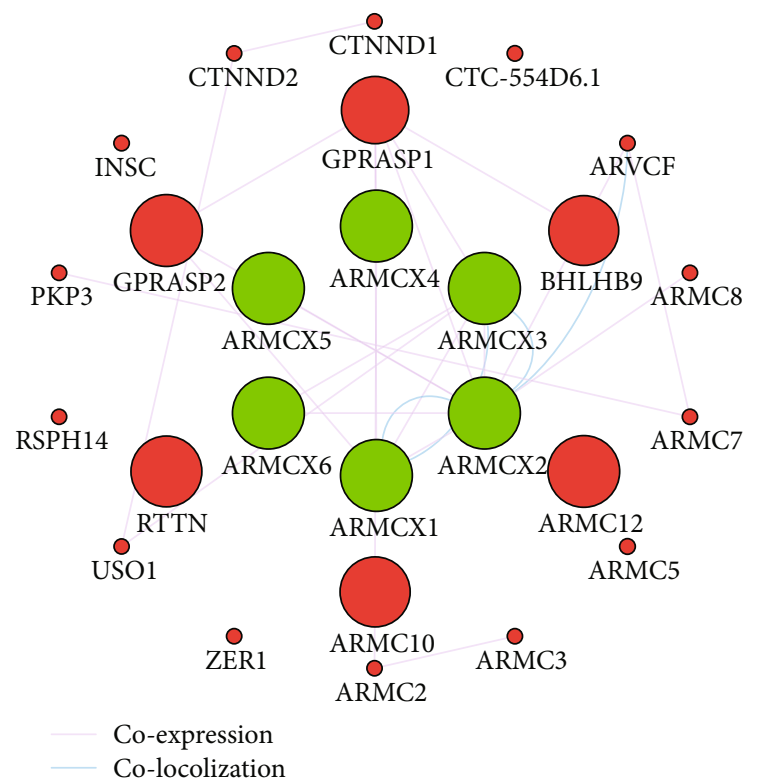

(b) (a)

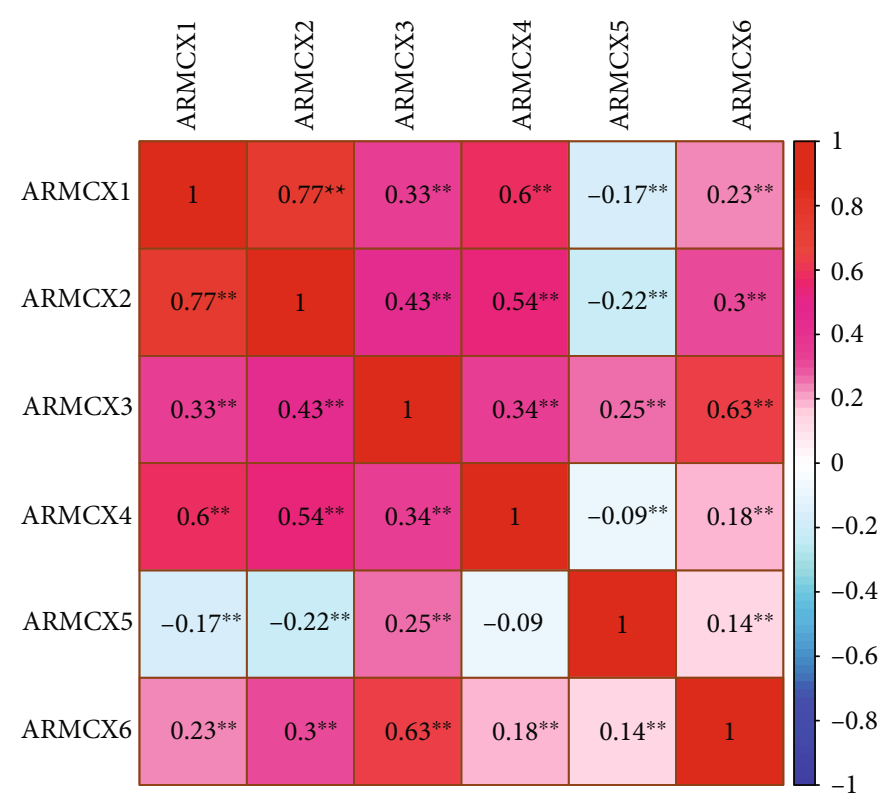

(c)

FIGURE 3: Functional assessment and bioinformatics analysis of ARMCX family genes (a). GeneMANIA constructed the gene-gene interaction network of the ARMCX family (b). Matrix graphs of Pearson's correlations of ARMCX family gene expression levels (c). ${ }^{* *} P<0.001$.

2.5. Joint-Effects Analysis. By analyzing the TCGA data, the results have shown that only ARMCX1 and ARMCX2 had statistical significance. The combination of ARMCX1 and ARMCX2 was investigated by joint-effects analysis. The combination included group 1 (low ARMCX1 and low ARMCX2 expression), group 2 (low ARMCX1 and high ARMCX2 expression, High ARMCX1 and low ARMCX2 expression), and group 3 (high ARMCX1 and high ARMCX2 expression). In addition, according to the results of TCGA database, age and tumor stage were adjusted in the Cox proportional hazards regression model.

2.6. Nomogram Model. Due to the clinical characteristics and risk score, a nomogram prediction model was constructed to evaluate the individual prognosis. Furthermore, the probable utility of the ARMCX family in predicting clinical grade was evaluated. In terms of clinical data and survival analysis, age, tumor stage, and ARMCX expression level were included in the risk model after Cox proportional risk regression model adjustment. Scores for each factor could be counted, and 1-year, 5-year, and 10-year survival rates also can be calculated [28].

2.7. Gene Set Enrichment Analysis. In order to explore the difference in biological functions and pathways in the survival of GC between low- and high-ARMCX gene expression groups, the potential mechanism in the molecular signature database (MSigDB) of c2 (c2.all.v6.1. Symbols) and c5 
TABLE 1: Clinical data characteristic of 351 GC patients.

\begin{tabular}{|c|c|c|c|c|c|}
\hline Items & Cases (total $n=351)$ & No. of events (\%) & MST (days) & Crude $P$ & Crude HR (95\% CI) \\
\hline \multicolumn{6}{|l|}{ Age } \\
\hline$\geq 60$ & 239 & $108(45.2)$ & 766 & \multirow{2}{*}{0.017} & Ref. \\
\hline$<60$ & 106 & $35(33.0 \%)$ & 1811 & & $0.629(0.429-0.923)$ \\
\hline Missing & 6 & & & & \\
\hline \multicolumn{6}{|l|}{ Gender } \\
\hline Male & 236 & $100(42.4 \%)$ & 869 & & Ref. \\
\hline Female & 125 & $44(35.2 \%)$ & 1043 & 0.184 & $0.787(0.552-1.122)$ \\
\hline Missing & 0 & & & & \\
\hline \multicolumn{6}{|c|}{ Tumor stage } \\
\hline $\mathrm{i}$ & 47 & $11(23.4 \%)$ & 2197 & & $0.260(0.126-0.537)$ \\
\hline ii & 109 & $34(31.2 \%)$ & 1686 & & $0.424(0.247-0.728)$ \\
\hline iii & 147 & $69(46.9 \%)$ & 779 & $<0.001$ & $0.643(0.397-1.042)$ \\
\hline iv & 35 & & & & \\
\hline Missing & 13 & $22(62.9 \%)$ & 476 & & Ref. \\
\hline
\end{tabular}

Abbreviations: MST: median survival time; Ref.: reference; HR: hazard ratio; 95\% CI: 95\% confidence interval.

TABLE 2: Univariate and multivariate survival analyses of the ARMCX family.

\begin{tabular}{|c|c|c|c|c|c|c|c|}
\hline Items & Cases (total $n=351$ ) & No. of events (\%) & MST (days) & Crude $P$ & Crude HR $(95 \% \mathrm{CI})$ & Adjusted $P$ & Adjusted HR (95\% CI) \\
\hline \multicolumn{8}{|c|}{ ARMCX1 } \\
\hline Low & 175 & $61(34.6 \%)$ & 1294 & \multirow{2}{*}{0.016} & $0.667(0.479-0.929)$ & \multirow{2}{*}{0.006} & $0.620(0.440-0.874)$ \\
\hline High & 176 & $83(47.6 \%)$ & 766 & & Ref. & & Ref. \\
\hline \multicolumn{8}{|c|}{ ARMCX2 } \\
\hline Low & 176 & $58(33.0 \%)$ & 1686 & \multirow{2}{*}{0.012} & $0.655(0.469-0.915)$ & \multirow{2}{*}{0.005} & $0.610(0.432-0.861)$ \\
\hline High & 175 & $86(49.1 \%)$ & 762 & & Ref. & & Ref. \\
\hline \multicolumn{8}{|c|}{ ARMCX3 } \\
\hline Low & 176 & $66(37.5 \%)$ & 1095 & \multirow{2}{*}{0.366} & Ref. & \multirow{2}{*}{0.690} & Ref. \\
\hline High & 175 & $78(44.6 \%)$ & 794 & & $1.163(0.838-1.616)$ & & $1.072(0.763-1.506)$ \\
\hline \multicolumn{8}{|c|}{ ARMCX4 } \\
\hline Lo & 176 & $70(39.8 \%)$ & 1095 & \multirow{2}{*}{0.570} & Ref. & \multirow{2}{*}{0.220} & Ref. \\
\hline High & 175 & $74(42.3 \%)$ & 801 & & $1.099(0.793-1.525)$ & & $1.238(0.880-1.741)$ \\
\hline \multicolumn{8}{|c|}{ ARMCX5 } \\
\hline Low & 176 & $76(43.2 \%)$ & 728 & \multirow{2}{*}{0.271} & Ref. & \multirow{2}{*}{0.507} & Ref. \\
\hline High & 175 & $68(38.9 \%)$ & 1153 & & $0832(0.599-1.155)$ & & $0.891(0.634-1.253)$ \\
\hline \multicolumn{8}{|c|}{ ARMCX6 } \\
\hline Low & 176 & $73(41.5 \%)$ & 1153 & \multirow{2}{*}{0.786} & Ref. & \multirow{2}{*}{0.929} & Ref. \\
\hline High & 175 & $71(40.6 \%)$ & 874 & & $0.956(0.689-1.326)$ & & $1.016(0.722-1.428)$ \\
\hline
\end{tabular}

Abbreviations: MST: median survival time; Ref.: reference; HR: hazard ratio; 95\% CI: 95\% confidence interval; Notes: Adjusted $P$, adjustment for age and tumor stage.

(c5.all.v6.1. Symbols) was studied by GSEA (http://software .broadinstitute.org/gsea/index.jsp, accessed Sept 27, 2019) [29-31].The nominal $P$ value $<0.05$ and the false discovery rate $(F D R)<0.25$ for the enriched gene sets in GSEA were statistically significant.

2.8. Statistical Analysis. Survival analysis was carried out by Kaplan-Meier and the log-rank test to calculate MSTs and $P$ values. The crude or adjusted HR and 95\% CI were calculated using the Cox proportional risk regression model for univariate and multivariate survival analyses. The Benjamini
Hochberg procedure was employed for multiple tests of FDR in GSEA to control [31-33], and $P<0.05$ was considered statistically significant. GraphPad Prism v.6.0 (La Jolla, CA) was used to draw vertical scatter plots and survival curves. SPSS software v.22.0 (IBM, Chicago, IL, USA) was employed for statistical analysis.

\section{Results}

3.1. ARMCX mRNA Expression Analysis. In human normal stomach tissue, ARMCX5 was expressed at a medium level 


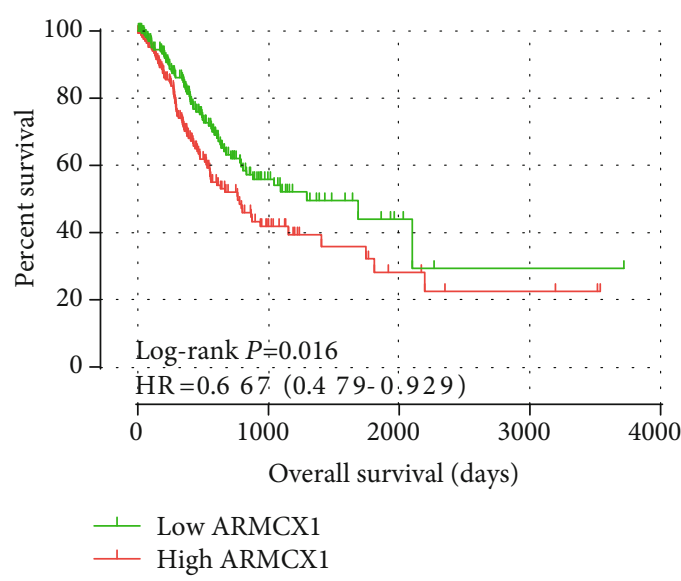

(a)

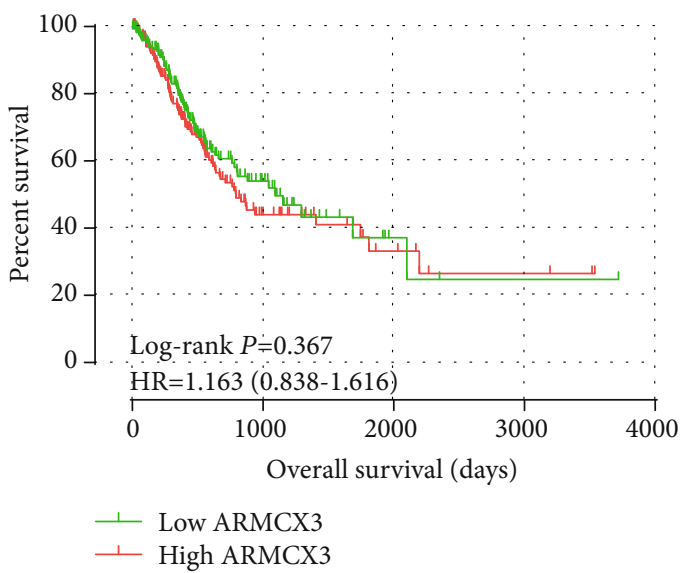

(c)

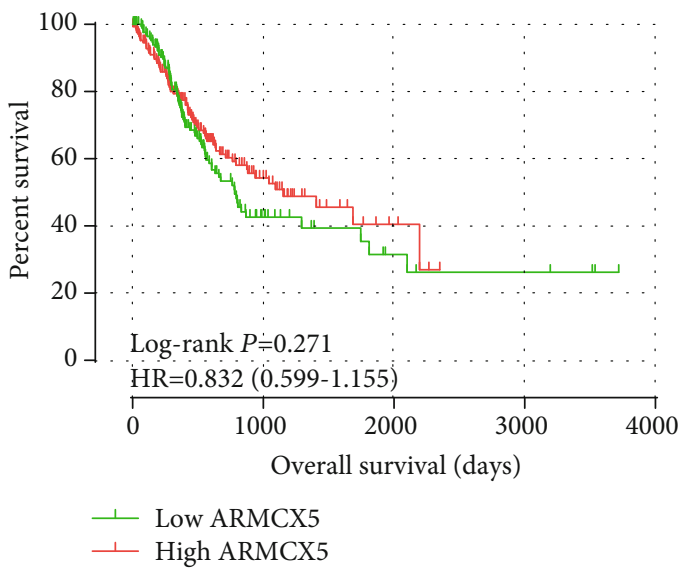

(e)

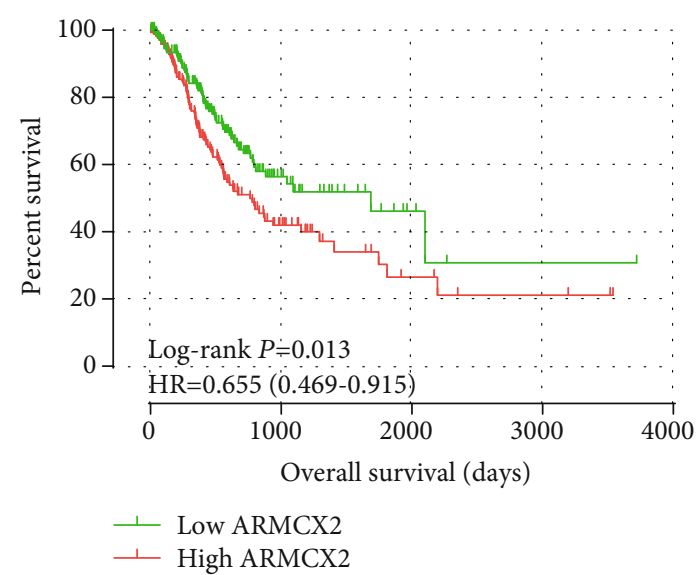

(b)

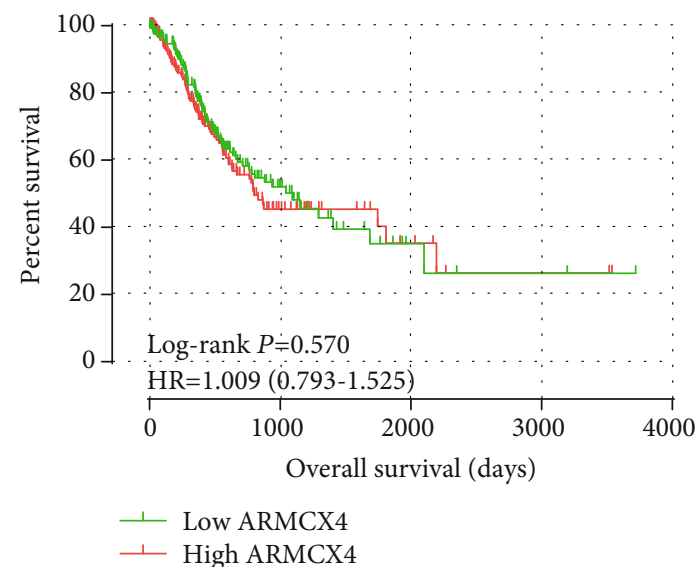

(d)

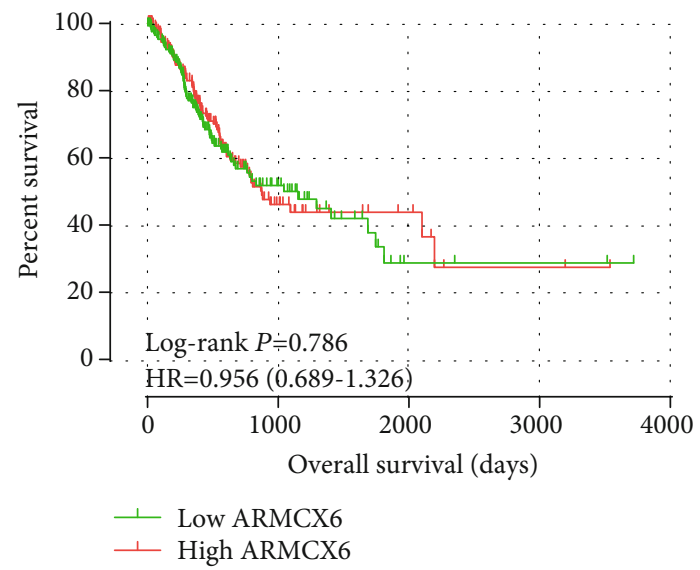

(f)

FIGURE 4: Univariate curves of OS of ARMCX family genes in GC. The lower expression levels of ARMCX1 and ARMCX2 were significantly associated with satisfactory OS results $(a, b)$. The expression of ARMCX3, ARMCX4, ARMCX5, and ARMCX6 mRNA did not have significant prognostic value for OS (c, d).

(Figure 1(e), whereas the other ARMCX genes (ARMCX1, ARMCX2, ARMCX3, ARMCX4, and ARMCX6) were expressed at low levels (Figures 1(a)-1(d) and 1(f)), compared with other normal tissues. Box plots of ARMCX1-6 genes were downloaded from GEPIA as shown in Figure 2.
ARMCX1, ARMCX2, and ARMCX4 were lowly expressed in primary gastric tumors and has a high expression in normal gastric tissues. However, conversely, ARMCX5 was less expressed in normal gastric tissues than in primary gastric tumors. The expression levels of ARMCX3 and ARMCX6 
have no significant difference between gastric tumors and normal gastric tissues.

3.2. Bioinformatics and Functional Annotation Analyses of the ARMCX Genes. Enrichment and functional analyses by KOBAS revealed that ARMCX genes were significantly enriched in ubiquitin ligase complex and the process of protein modification (Figure 3(a)). However, we have not found any associations of the ARMCX family using Kyoto Encyclopedia of Genes and Genomes (KEGG) and Database for Annotation, Visualization, and Integrated Discovery (DAVID) analyses. By analyzing gene-gene and proteinprotein interaction networks, we confirmed that the ARMCX family had strong protein homology and coexpression at both gene and protein levels, as shown in Figure 3(b).

3.3. Correlation Analysis Value Assessment of the ARMCX Family. Coexpression analyses of individual ARMCX genes were analyzed using Pearson's correlation coefficient. The expression level of ARMCX1, ARMCX2, ARMCX3, and ARMCX6 was correlated with each other. Furthermore, there was no significant correlation between the expressions of ARMCX4 and ARMCX5, but both the expressions of ARMCX4 and ARMCX5 all related to the other members of the ARMCX family $\left({ }^{* *} P<0.01\right.$; Figure $\left.3(\mathrm{c})\right)$.

3.4. Clinical Characteristics of GC Patients. There were 351 GC patients who had prognosis information included in the current study; UCSC Xena dataset is shown in Table 1. The univariable survival analysis revealed that age and tumor stage were correlated with MST in combination with clinical data $(P=0.017$ and $P<0.001$, respectively), and preliminary stage was significantly correlated with favorable MST (2197 days, $P<0.001, \mathrm{HR}=0.260$, $95 \% \mathrm{CI}=0.126-0.537)$. On the other hand, gender was not associated with MST.

3.5. Survival Analysis of the ARMCX Gene Family. Survival analysis is shown in Table 2 and Figure 4. Due to the age and tumor stage that were related with MST, both age and tumor stage were analyzed using the multivariate Cox proportional risk regression model. In univariate survival analysis, lower expression levels of ARMCX1 and ARMCX2 were significantly associated with satisfactory OS results (log-rank $P=0.016, \quad \mathrm{HR}=0.667, \quad 95 \% \mathrm{CI}=0.479-0.929$; log-rank $P=0.013, \quad H R=0.655, \quad 95 \% C I=0.469-0.915$, respectively; Figures $4(\mathrm{a})$ and $4(\mathrm{~b}))$. The expression of ARMCX3, ARMCX4, ARMCX5, and ARMCX6 mRNA did not have a significant prognostic value for OS (logrank $P=0.367,0.570,0.271$, and 0.786 , respectively; Figures $4(\mathrm{c})-4(\mathrm{f}))$.

3.6. Joint-Effects Analysis of ARMC1 and ARMCX2. Based on the findings in the multivariate survival analysis, ARMCX1 and ARMCX2 were associated with a significantly different survival. A joint-effects analysis was employed to further determine the combined effects in prognostic prediction of ARMCX1 and ARMCX2 (grouped as summarized in Table 3). The combination of ARMCX1 and ARMCX2 included group 1, group 2 and group 3, and results are
TABLE 3: Grouping information for joint-effects analysis.

\begin{tabular}{lc}
\hline Group & Combinations \\
\hline 1 & Low ARMCX1+low ARMCX2 \\
2 & Low ARMCX1+high ARMCX2 \\
3 & High ARMCX1+low ARMCX2 \\
\hline
\end{tabular}

Abbreviations: ARMCX: arm protein lost in epithelial cancers, $\mathrm{X}$ chromosome.

shown in Table 4. Group 1 had the longest MST of 1686 days (adjusted $P=0.003$ ), while group 3 had the shortest MST of 762 days (adjusted $P=0.012$ ). Kaplan-Meier survival analyses of ARMCX1 and ARMCX2 are shown in Figure 5. Low expression levels of ARMCX1 and ARMCX2 in group 1 were significantly correlated with better clinical outcome. In group 3, high expression of ARMCX1 and ARMCX2 was correlated with poor OS (log-rank $P=0.007)$.

3.7. Nomogram Model. Nomogram risk scoring includes age, tumor stage, and the expression level of ARMCX1 and ARMCX2 to calculate 1-year, 5-year, and 10-year related survival rates. The higher total points, the lower survival rate, and the results substantiated that high expression levels of ARMCX1 and ARMCX2, age of the patient ( $>60$ years old), and advanced tumor stage established a prognostic feature that conduced to the highest risk for poor OS (Figure 6).

3.8. Gene Set Enrichment Analysis. In order to further explore the underlying mechanisms of ARMCX genes in GC prognosis, we used the PAAD genome-wide RNA sequencing dataset for GSEA. GSEA results of the $c 2$ reference gene set revealed that a low ARMCX1 expression was involved in the WNT signaling pathway, regulation of cell metastasis (Figure $7(\mathrm{a})$ ) and cell cycle biological processes (Figures $7(\mathrm{~b})-7(\mathrm{~h})$ ), and poor survival of lung cancer (Figure $7(\mathrm{i})$ ). Also, the enrichment of $\mathrm{c} 5$ indicates that low ARMCX1 is also involved in cell division (Figure 8(c)), cell cycle (Figures 8(a) and $8(\mathrm{~b})$ ), gene silencing (Figure $8(\mathrm{~d})$ ), RNA modification (Figure 8(i)), and NF-kappaB signaling pathway (Figures $8(\mathrm{~g})$ and $8(\mathrm{~h})$ ). GSEA results of $\mathrm{c} 2$ enrichments reveal that the low expression of ARMCX2 was correlated to the cell cycle biological process (Figures 9(a), 9(b), and 9(f)), regulation of apoptosis (Figure $9(\mathrm{~h})$ ), DNA replication (Figure 9(c)) and damage response (Figure 9(g)), and E2F, WNT, and NF-kappaB signaling pathways (Figures 9(d), $9(\mathrm{e})$, and $9(\mathrm{e}))$, whereas the $\mathrm{c5}$ enrichments suggest that low ARMCX2 expression is involved in the biological process of cell division (Figure 10(d)), cell cycle (Figures 10(b), 10(c), and 10(i)), apoptosis (Figure 10(e)), gene silencing (Figure 10(g)), DNA damage checkpoint (Figure 10(f)), and the NF-kappaB signaling pathway (Figure 10(a)). Moreover, the remaining results of this study can be seen in Supplementary Tables 1 and 1 . 
TABLE 4: Joint-effects analysis of the combination of ARMCX1 and ARMCX2.

\begin{tabular}{lccccccc}
\hline Items & Cases (total $n=351)$ & No. of events (\%) & MST (days) & Crude $P$ & Crude HR (95\% CI) & Adjusted $P$ & Adjusted HR (95\% CI) \\
\hline Group 1 & 143 & $46(32.2 \%)$ & 1686 & 0.007 & $0.600(0.414-0.870)$ & 0.003 & $0.563(0.384-0.825)$ \\
Group 2 & 65 & $27(41.5 \%)$ & 766 & 0.549 & $0.873(0.559-1.362)$ & 0.506 & $0.854(0.537-1.358)$ \\
Group 3 & 143 & $71(49.7 \%)$ & 762 & 0.025 & Ref. & 0.012 & Ref. \\
\hline
\end{tabular}

Abbreviations: MST: median survival time; Ref.: reference; HR: hazard ratio; 95\% CI: 95\% confidence interval; Notes: Adjusted $P$, adjustment for age and tumor stage.

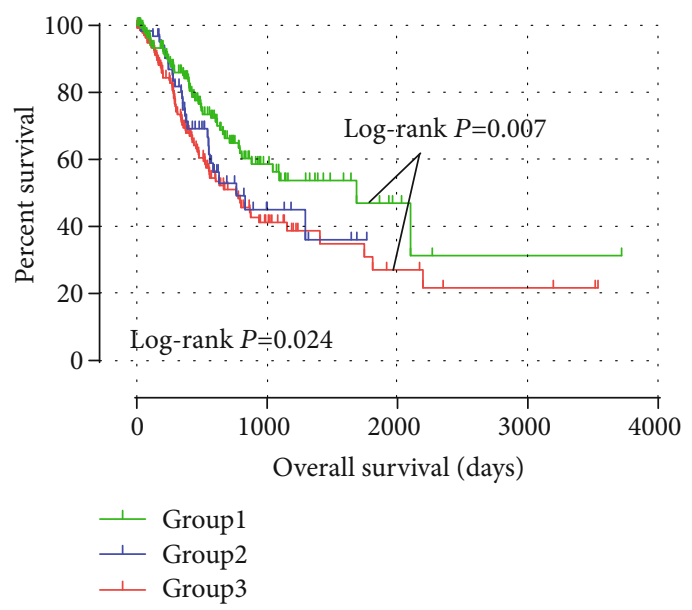

FIgURE 5: Survival curves for joint-effects analysis of the combination of ARMCX1 and ARMCX2 genes in the TCGA database. Lowexpression levels of ARMCX1 and ARMCX2 in group 1 were significantly correlated with better clinical outcome. In group 3, high expression of ARMCX1 and ARMCX2 was correlated with poor OS.

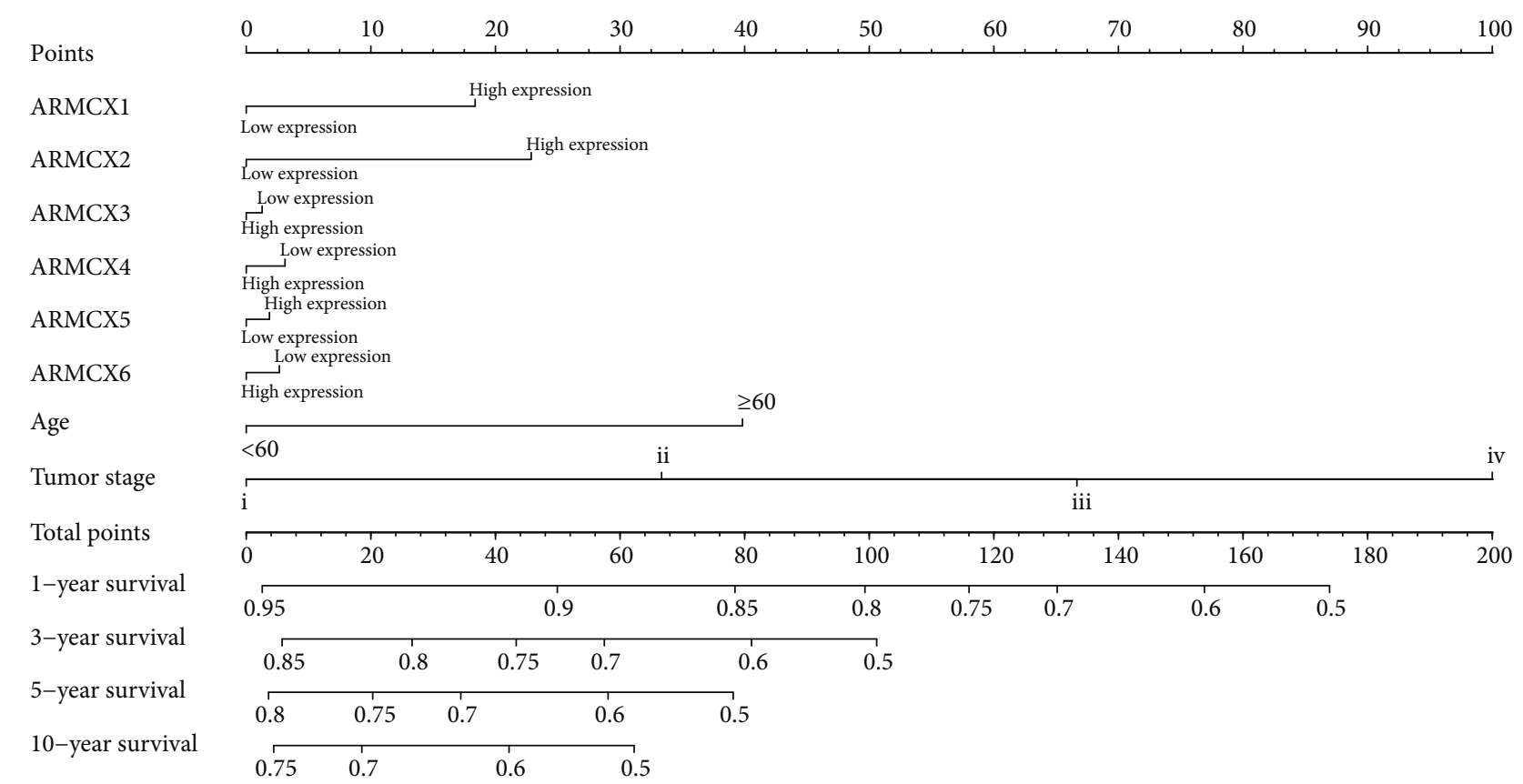

FIGURE 6: Nomogram for predicting the 1-, 3-, 5-, and 10-year events (death) with risk scores and clinical parameters. The high expression levels of ARMCX1 and ARMCX2, age of the patient ( $>60$ years old), and advanced tumor stage established a prognostic feature that conduced to the highest risk for poor OS. 


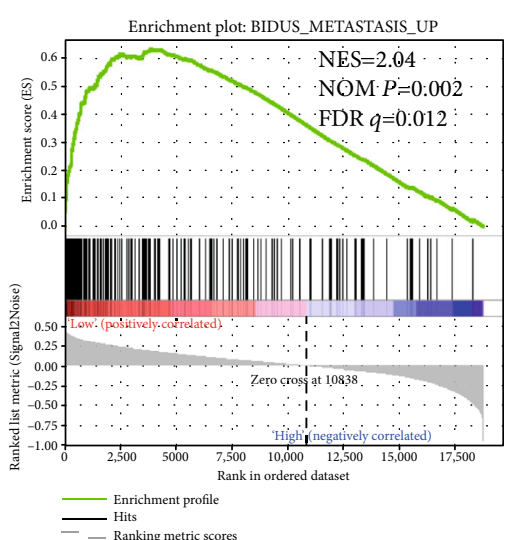

(a)

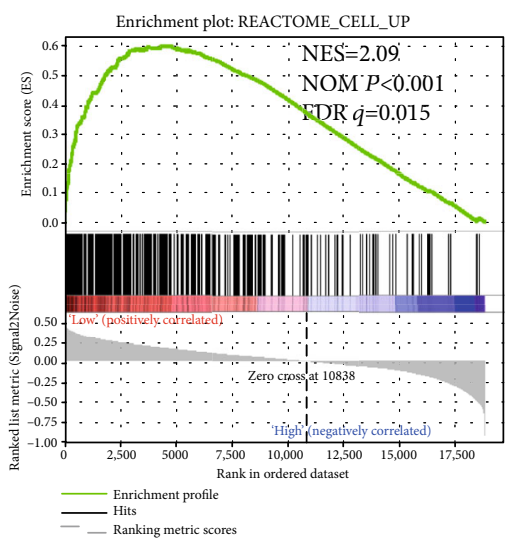

(d)

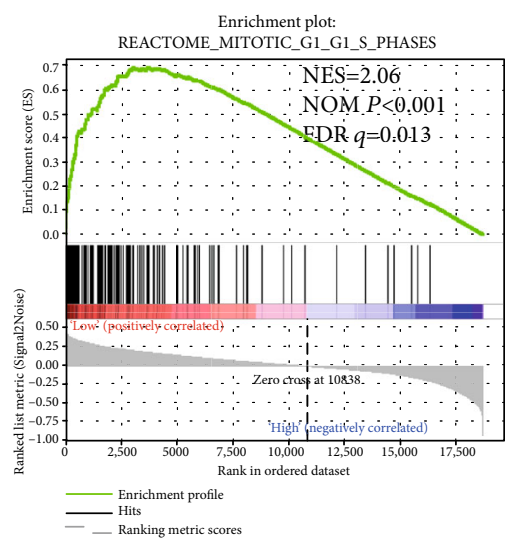

(g)

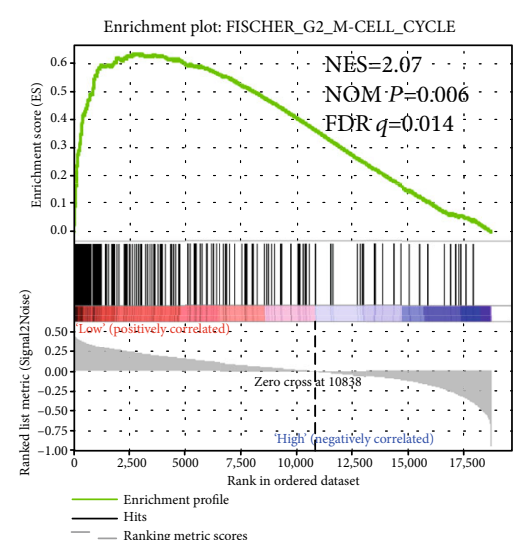

(b)

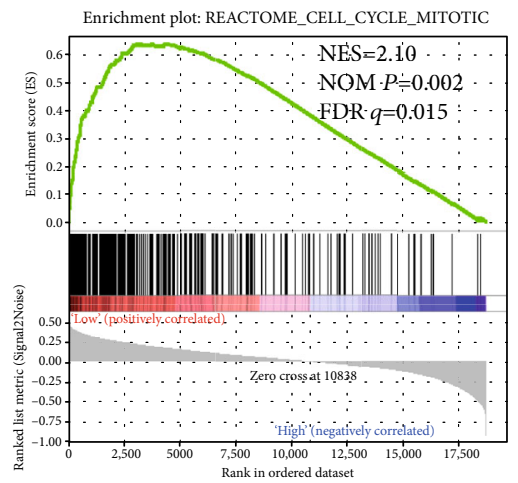

— $\begin{gathered}\text { Enrichment profile } \\ \text { Hits } \\ \text { Ranking metric scores }\end{gathered}$

(e)

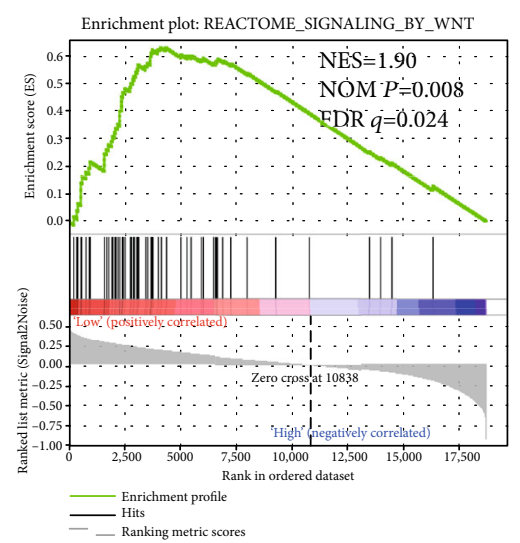

(h)

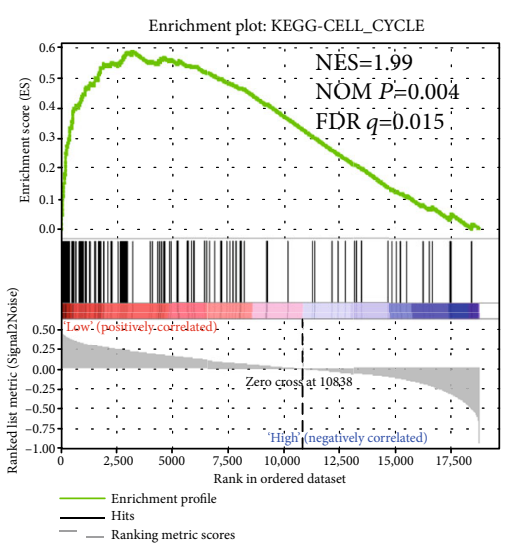

(c)
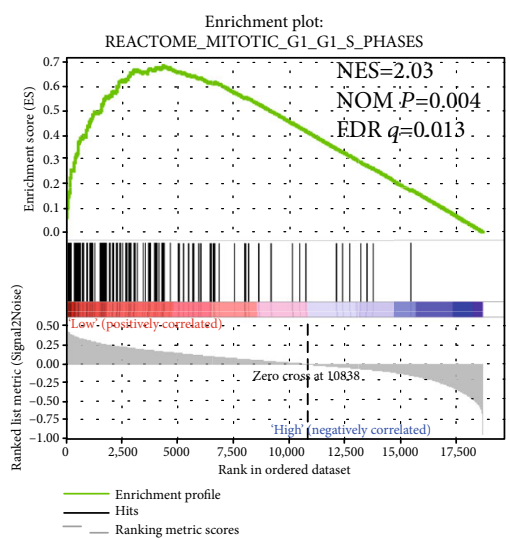

(f)

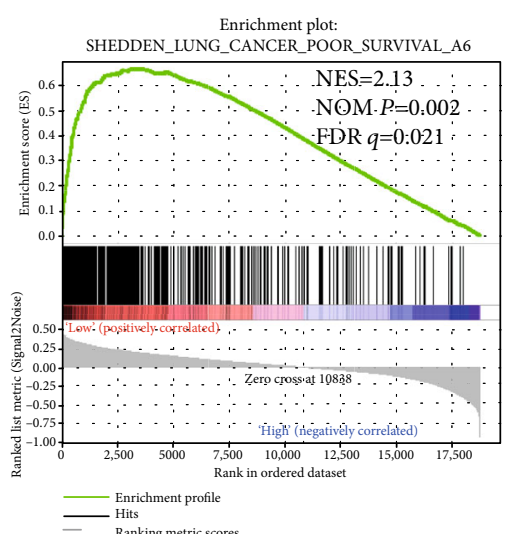

(i)

FIGURE 7: GSEA results of low ARMCX1 expressed in GC patients, using gene set c2. The low ARMCX1 expression was involved in the WNT signaling pathway, regulation of cell metastasis (a) and cell cycle biological processes (b-h), and poor survival of lung cancer (i). Abbreviations: FDR: false discovery rate; GSEA: gene set enrichment analysis; KEGG: Kyoto Encyclopedia of Genes and Genomes; NES: normalized enrichment score; NOM: nominal.

\section{Discussion}

In our present study, we elucidated the associations between the expression levels of ARMCX 1-6 genes with the prognosis of GC patients. Our research disclosed that ARMCX 1 and ARMCX 2 contribute significantly to OS, but ARMCX 3-6 show no significant association with OS. Thus, the expression levels of ARMCX 1 and ARMCX
2 both alone and in combination may serve as potential biomarkers of GC.

In 1989, the armadillo family proteins were first discovered in the polar gene fragment of Drosophila [3]. Subsequently, more and more proteins containing arm repeats have been analyzed and sequenced. Armadillo repeats containing $\mathrm{x}$-chain (ARMCX 1-6) are involved in many biological processes, such as mediating protein-protein interactions 


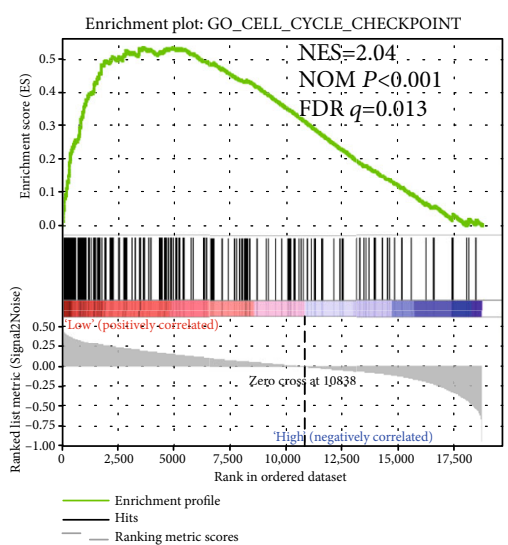

(a)

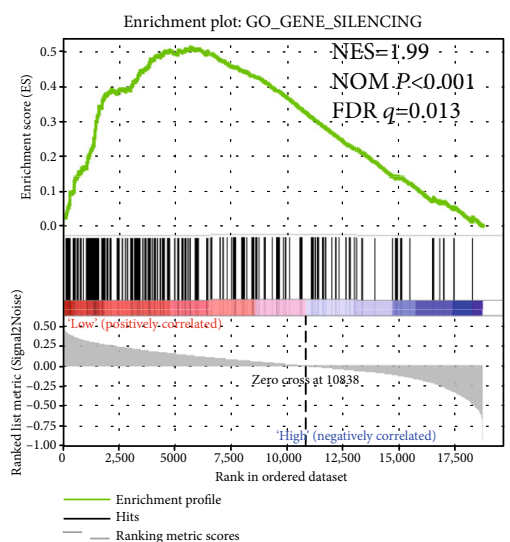

(d)

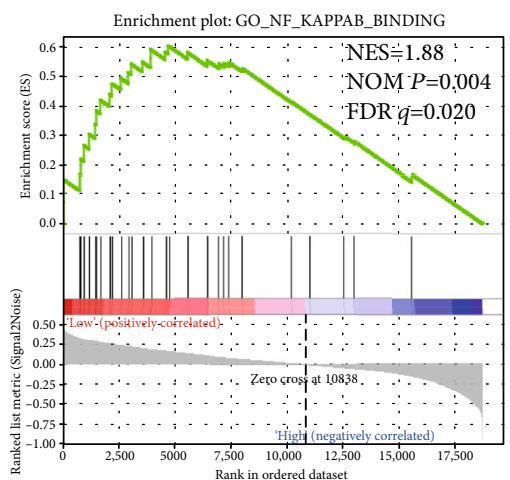

三 $\begin{gathered}\text { Enichment profle } \\ \text { Hits }\end{gathered}$

(g)

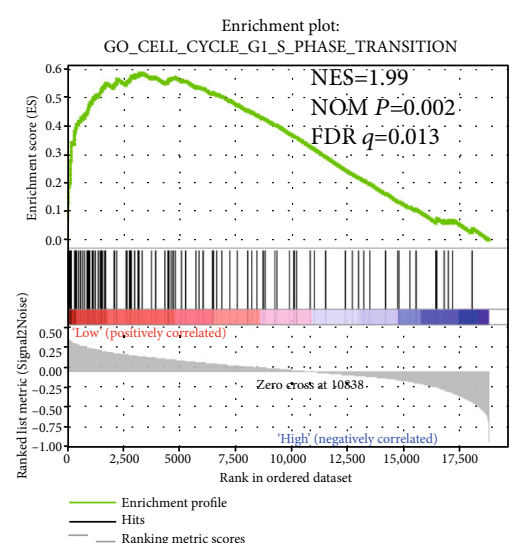

(b)
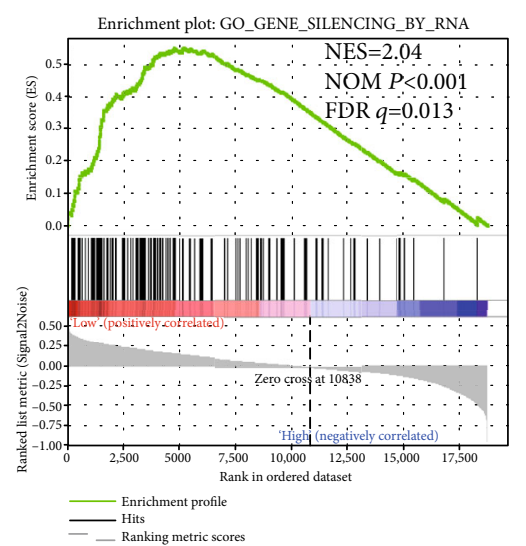

(e)

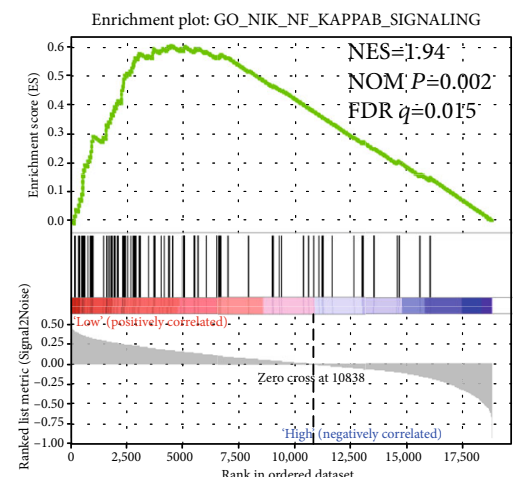

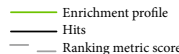

(h)

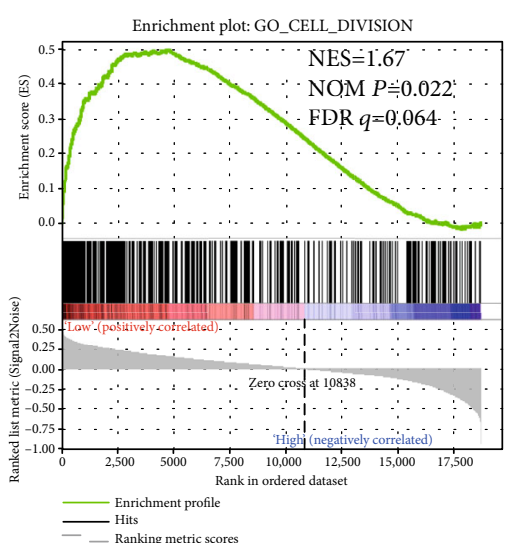

(c)

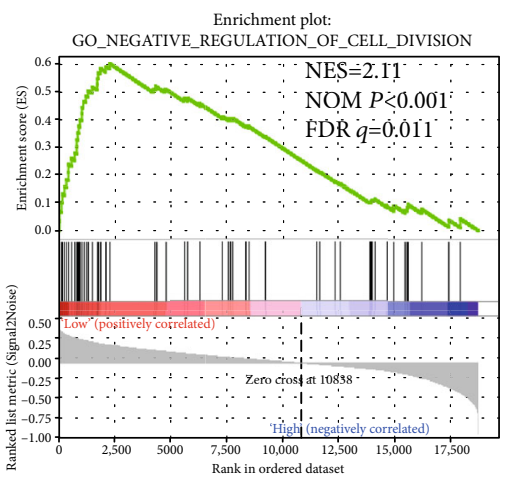

—— Enrichment profile

(f)
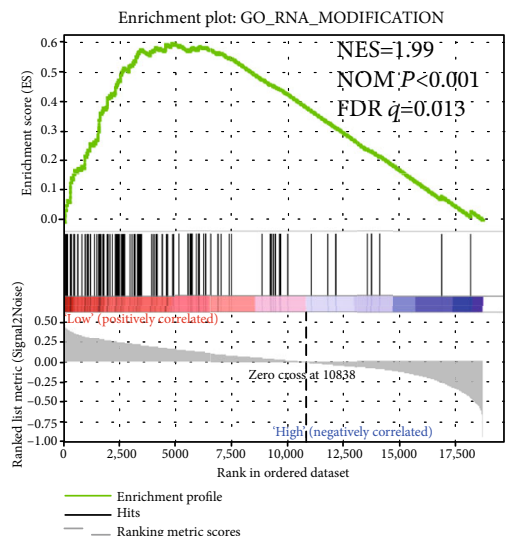

(i)

FIGURE 8: GSEA results of low ARMCX1 expressed in GC patients, using gene set c5. The low ARMCX1 is also involved in cell division (c), cell cycle ( $a, b)$, gene silencing (d), RNA modification (i), and the NF-kappaB signaling pathway ( $\mathrm{g}, \mathrm{h})$. Abbreviations: FDR: false discovery rate; GSEA: gene set enrichment analysis; KEGG: Kyoto Encyclopedia of Genes and Genomes; NES: normalized enrichment score; NOM: nominal.

and intervening in cell assembly, nuclear transport, and transcriptional activation [34]. Many studies have demonstrated that ARMCX is associated with the risk and prognosis of several diseases. For instance, the ARMCX family plays an important role in embryogenesis and tumorigenesis [22]. Scholars have found that some members of the ARMCX protein family (Armcx1-3) were underexpressed in several cancers of epithelial origin, including the lung, prostate, colon, and pancreatic [11].
ARMCX1, ARMCX2, and ARMCX3 are located in the chromosome region xq21.33-q22.2, respectively. Their amino $\mathrm{N}$-terminal region has a transmembrane domain, indicating that these proteins may be located in the membrane structure of cells. ARMCX3 has been found to be a complete membrane protein of the mitochondrial outer membrane, which functions by interacting with transcription regulator Sox10 [12]. In addition, ARMCX4, ARMCX5, and ARMCX6 were located in chromosome regions xq22.1, 


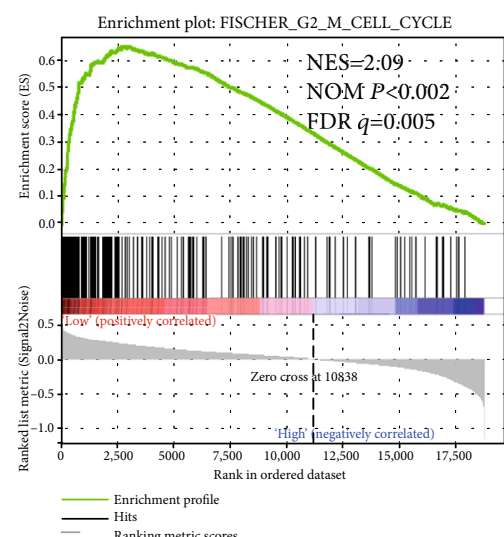

(a)

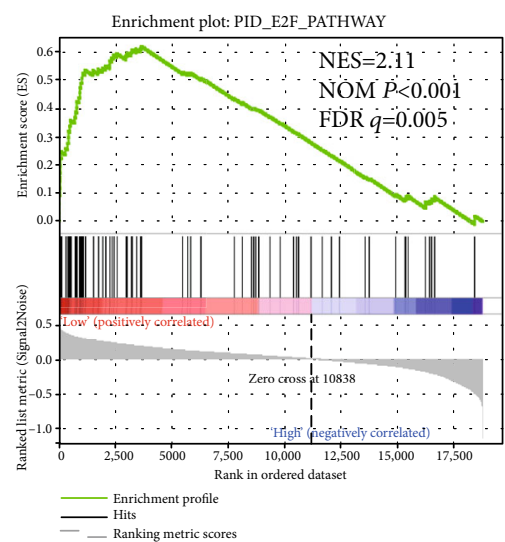

(d)

REACTOME_P53_DEPENDENT_GI__DBA_DAMAGE_RESPONSE

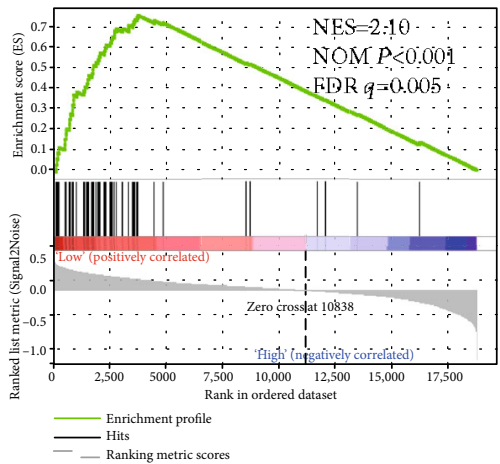

(g)

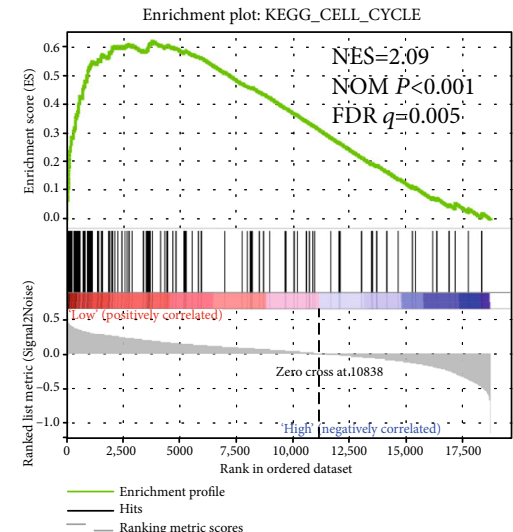

(b)
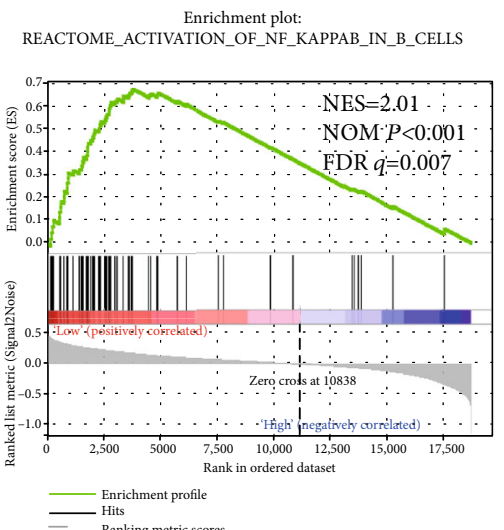

(e)

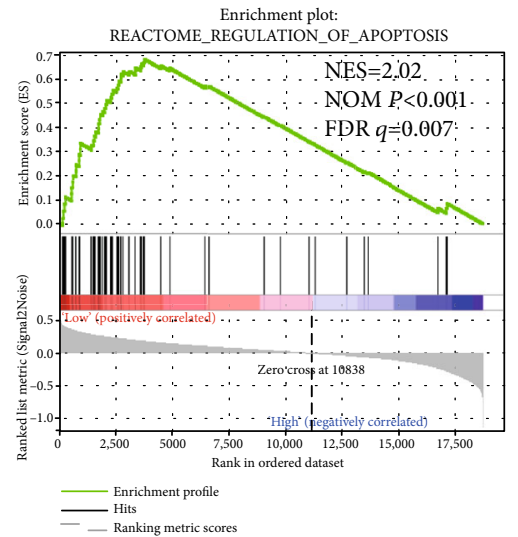

(h)

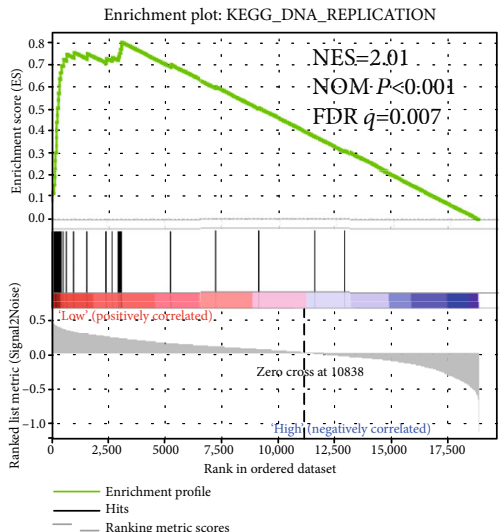

(c)

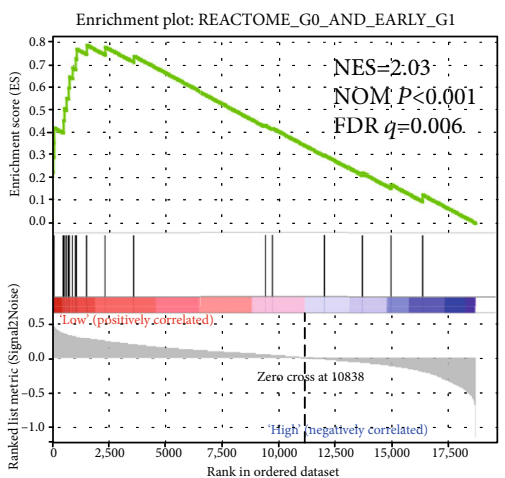

- Enrichment profile

(f)

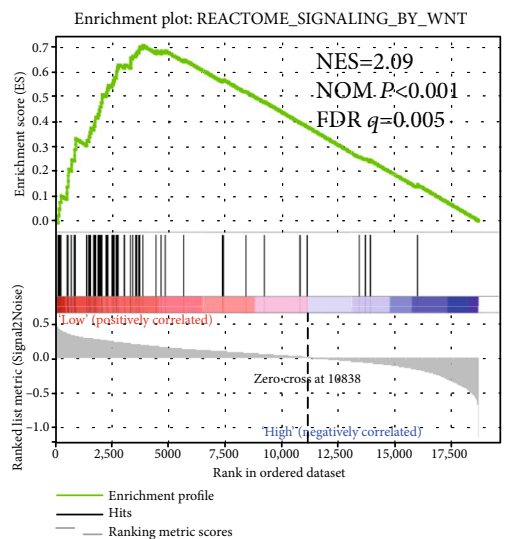

(i)

FIgURE 9: GSEA results of low ARMCX2 expressed in GC patients, using gene set c2. The low expression of ARMCX2 was correlated to the cell cycle biological process (a, b, and f), regulation of apoptosis (h), DNA replication (c) and damage response (g), and the E2F, WNT, and NF-kappaB signaling pathways (d, i, and e). Abbreviations: FDR: false discovery rate; GSEA: gene set enrichment analysis; KEGG: Kyoto Encyclopedia of Genes and Genomes; NES: normalized enrichment score; NOM: nominal.

xq22.1-q22.3, and xq21.33-q22.3, respectively. Studies have shown that ARMCX5 can be activated by binding to the oncogene $\mathrm{ZnF} 217$ [35] and ARMCX6 upexpressed at least 2 -fold in peripheral blood monocytes of rheumatoid arthritis patients compared to those identified using oligonucleotide array [36]. Moreover, regardless of their function in other diseases, they are associated with tumorigenesis and were initially described as presumed tumor suppressors [11].
Here, we downloaded and analyzed data from GEO online database to determine the potential relationship between ARMCX mRNA expression and clinical outcomes of patients with gastric cancer. We observed significant differences in the expression of ARMCX1, ARMCX2, ARMCX4, and ARMCX5 between primary tumors and adjacent normal tissues, without ARMCX3 and ARMCX6. More importantly, ARMCX1 and ARMCX2 are more highly expressed in adjacent normal 


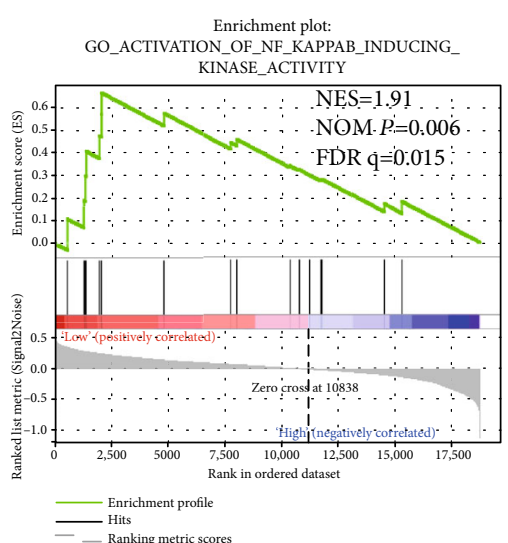

(a)

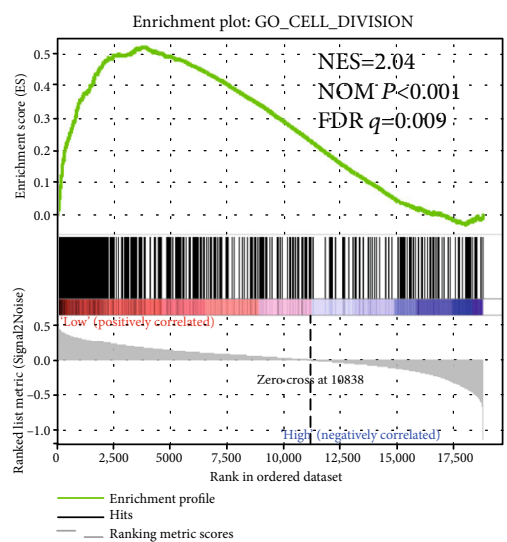

(d)

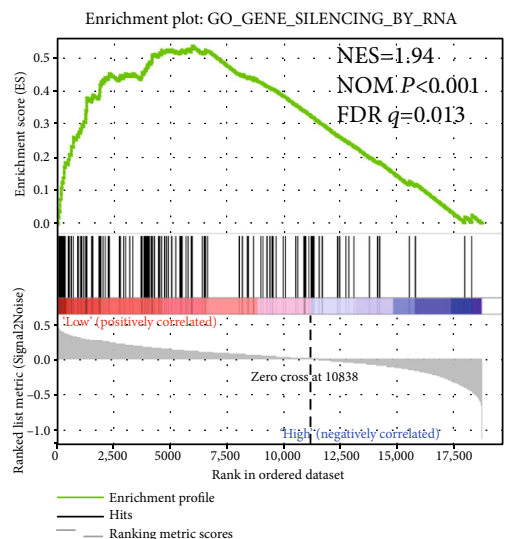

(g)

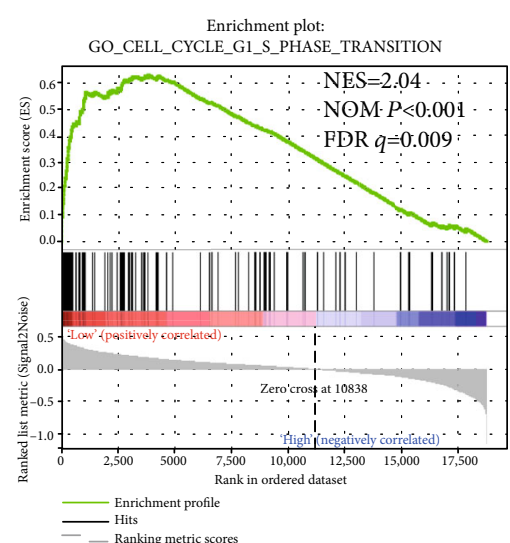

(b)

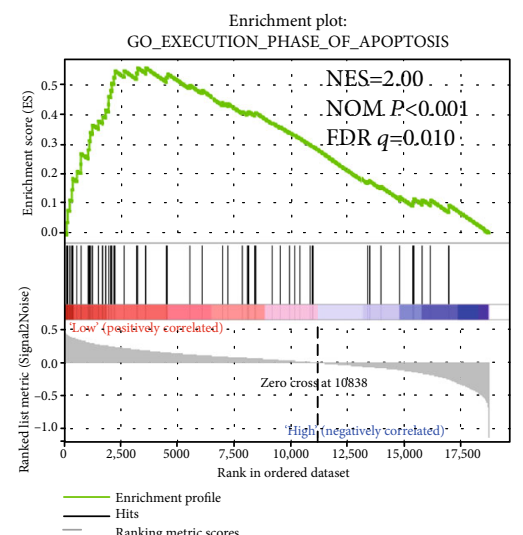

(e)

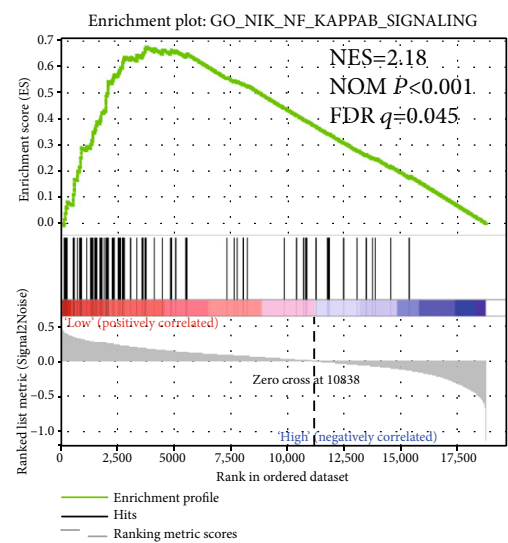

(h)

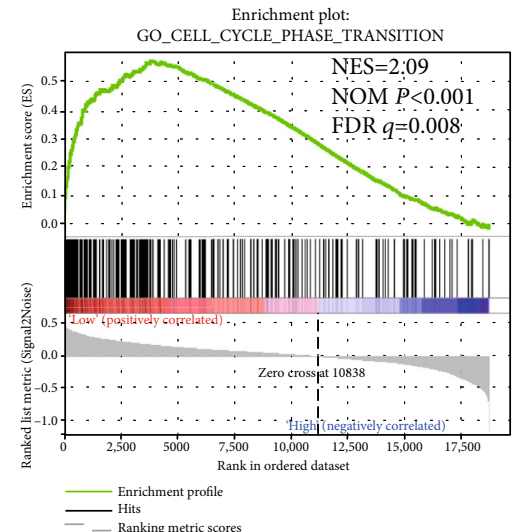

(c)

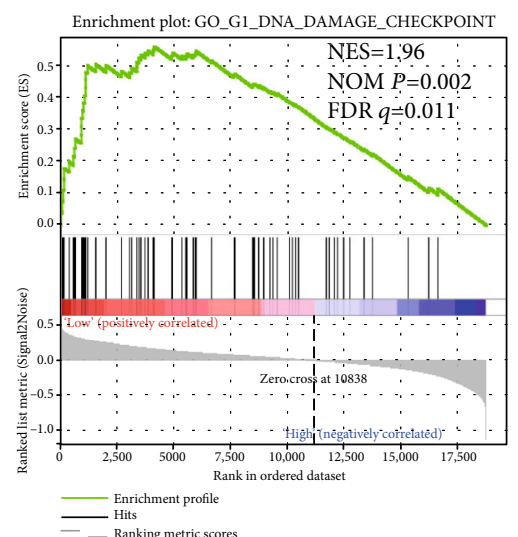

(f)

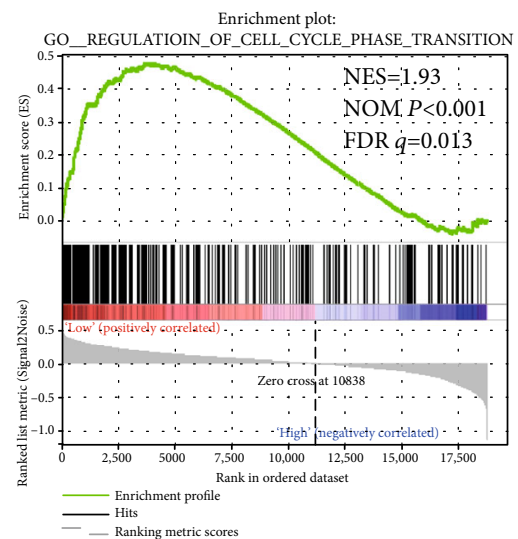

(i)

FIgURE 10: GSEA results of low ARMCX2 expressed in GC patients, using gene set c5. The low ARMCX2 expression is involved in biological process of cell division (d), cell cycle (c, i), apoptosis (e), gene silencing (g), DNA damage checkpoint (f), and the NF-kappaB signaling pathway (a). Abbreviations: FDR: false discovery rate; GSEA: gene set enrichment analysis; KEGG: Kyoto Encyclopedia of Genes and Genomes; NES: normalized enrichment score; NOM: nominal.

tissues than in tumor tissues, leading to better OS in patients with gastric cancer, although the mechanism of action needs further clarification.

In addition, a comprehensive survival analysis of the current prognostic characteristics of ARMCX was performed by establish a nomogram, and stratified joint-effects survival analysis was conducted to explore its potential application.
The results indicated that high ARMCX expression was an independent risk factor as a prognostic characteristic for patients with gastric cancer, and the relevant risk score could be used as a prognostic indicator. Nomogram, composed of risk score and other clinical information such as age and tumor stage, is an important prognostic risk assessment system for gastric cancer. 
To explore the underlying mechanism of ARMCX genes in gastric cancer prognosis, we used a genome-wide RNA sequencing dataset in GSEA. The NF-kappaB, E2F and WNT signaling pathways, the cell cycle, and gene silencing were significantly enriched in the ARMCX1 and ARMCX2 low-expression groups.

It is well established that, as members of the armadillo (Arm) family, $\beta$-catenin and adenomatous polyposis coli (APC) are important components of the WNT signaling pathway. Moreover, WNT signaling plays an important role in a variety of biological processes, such as tumorigenesis, embryonic development, and stem cell maintenance [37-39]. $\beta$-Catenin, which is a multifunctional protein, plays an essential role in a variety of biological responses. For instance, in the WNT signaling pathway, $\beta$-catenin works by interact with E-cadherin and TCF/LEF transcription factors, respectively $[40,41]$. APC can regulate the WNT signaling pathway by synergistically acting with casein kinases 1 , glycogen synthase kinase-3b, and AXIN to induce degradation of $\beta$-catenin $[37,42,43]$. On the basis of GSEA results, we deduced that both ARMCX1 and ARMCX2 were involved in the pathway and biological processes that are associated with the progress and treatment of gastric cancer and may serve as a GC prognostic marker. Once these results are verified, ARMCX1 and ARMCX2 may be used as biomarkers in combination with other clinical factors to facilitate the selection of diagnosis and treatment decisions for GC and to benefit patients with better clinical outcomes.

Although significant results have been achieved in the current study, there are still some deficiencies to be considered. First, the results of this study were obtained from a single cohort in the TCGA database, and its demographic characteristics may not be representative of all patient groups. Therefore, genetic changes may have deviation and require further validation in other GC groups. Second, the clinical information from TCGA was incomplete. Therefore, we cannot conduct a comprehensive stratification analysis in the Cox proportional risk regression model including all layers. Third, the mechanism between the above ARMCX and WNT signaling pathways affecting the clinical prognosis of GC still needs to be determined.

\section{Conclusion}

Our present study has determined that ARMCX has a potential prognostic value for gastric cancer and may have clinical application value. In addition, further basic research is needed to clarify the specific mechanisms of ARMCX in GC.

\section{Data Availability}

The datasets used and/or analyzed during the current study are available from the corresponding author on reasonable request and TCGA data base.

\section{Ethical Approval}

This article does not contain any studies with human participants or animals performed by any of the authors. Since all datasets included in the present study were downloaded from TCGA, additional approval by an Ethics Committee was not needed. The procedures were in accordance with the Helsinki declaration of 1964 and its later amendments.

\section{Conflicts of Interest}

The authors declare that they have no conflicts of interest.

\section{Authors' Contributions}

TW, YQ, and XL conceived and designed the study. $\mathrm{ZH}$, WW, and ZL interpreted the data and performed the analysis. TW wrote the article, and $\mathrm{MH}$ approved the final version of the manuscript. All authors approved the final version of the manuscript. TingAn Wang and HuaGe Zhong contributed equally to this study.

\section{Acknowledgments}

We sincerely appreciate TCGA (https://cancergenome.nih .gov/) and UCSC Xena (http://xena.ucsc.edu/) for sharing the GC data and for Dr Liucheng Wu for his advice with the language consulting of the manuscript. The study was supported by the National Natural Science Foundation of GuangXi (No. 2019GXNSFBA185019) and National Natural Science Foundation of China (No. 81760521).

\section{Supplementary Materials}

Supplementary 1. Supplementary Table 1: GSEA KEGG (c2.all.v6.2.symbols.gmt) results of ARMCX1 and ARMCX2.

Supplementary 2. Supplementary Table 2: GSEA GO (c5.all.v6.2.symbols.gmt) functional analysis results of ARMCX1 and ARMCX2.

\section{References}

[1] E. Van Cutsem, X. Sagaert, B. Topal, K. Haustermans, and H. Prenen, "Gastric cancer," The Lancet, vol. 388, no. 10060, pp. 2654-2664, 2016.

[2] R. L. Siegel, K. D. Miller, and A. Jemal, "Cancer statistics, 2019," CA: a Cancer Journal for Clinicians, vol. 69, no. 1, pp. 7-34, 2018.

[3] B. Riggleman, E. Wieschaus, and P. Schedl, "Molecular analysis of the armadillo locus: uniformly distributed transcripts and a protein with novel internal repeats are associated with a Drosophila segment polarity gene," Genes \& Development, vol. 3, no. 1, pp. 96-113, 1989.

[4] G. López-Doménech, R. Serrat, S. Mirra et al., "The Eutherian Armcx genes regulate mitochondrial trafficking in neurons and interact with Miro and Trak2," Nature Communications, vol. 3, no. 1, p. 814, 2012.

[5] M. Peifer, S. Berg, and A. B. Reynolds, "A repeating amino acid motif shared by proteins with diverse cellular roles," Cell, vol. 76, no. 5, pp. 789-791, 1994. 
[6] M. A. Andrade, C. Petosa, S. I. O’Donoghue, C. W. Müller, and P. Bork, "Comparison of ARM and HEAT protein repeats"," Journal of Molecular Biology, vol. 309, no. 1, pp. 1-18, 2001.

[7] A. H. Huber, W. J. Nelson, and W. Weis, "Three-Dimensional Structure of the Armadillo Repeat Region of $\beta$-Catenin," Cell, vol. 90, no. 5, pp. 871-882, 1997.

[8] H. J. Choi and W. I. Weis, "Structure of the armadillo repeat domain of plakophilin 1," Journal of Molecular Biology, vol. 346, no. 1, pp. 367-376, 2005.

[9] H. Striegl, M. A. Andrade-Navarro, and U. Heinemann, "Armadillo motifs involved in vesicular transport," PLoS One, vol. 5, no. 2, article e8991, 2010.

[10] F. Parmeggiani, R. Pellarin, A. P. Larsen et al., "Designed Armadillo Repeat Proteins as General Peptide-Binding Scaffolds: Consensus Design and Computational Optimization of the Hydrophobic Core," Journal of Molecular Biology, vol. 376, no. 5, pp. 1282-1304, 2008.

[11] I. V. Kurochkin, N. Yonemitsu, S. I. Funahashi, and H. Nomura, "ALEX1, a Novel Human Armadillo Repeat Protein That Is Expressed Differentially in Normal Tissues and Carcinomas," Biochemical and Biophysical Research Communications, vol. 280, no. 1, pp. 340-347, 2001.

[12] Z. Mou, A. R. Tapper, and P. D. Gardner, "The armadillo repeat-containing protein, ARMCX3, physically and functionally interacts with the developmental regulatory factor Sox10," The Journal of Biological Chemistry, vol. 284, no. 20, pp. 13629-13640, 2009.

[13] C. A. Smith, P. J. McClive, and A. H. Sinclair, "Temporal and spatial expression profile of the novelarmadillo-related gene,Alex2, during testicular differentiation in the mouse embryo," Developmental Dynamics, vol. 233, no. 1, pp. 188193, 2005.

[14] F. Zeng, K. Liao, J. Wu et al., "ALEX1 may be a novel biomarker for human cervical squamous cell carcinoma," International Journal of Clinical and Experimental Pathology, vol. 8, no. 8, pp. 9434-9439, 2015.

[15] H. Iseki, A. Takeda, T. Andoh et al., "ALEX1 suppresses colony formation ability of human colorectal carcinoma cell lines," Cancer Science, vol. 103, no. 7, pp. 1267-1271, 2012.

[16] C. Montavon, B. S. Gloss, K. Warton et al., "Prognostic and diagnostic significance of DNA methylation patterns in high grade serous ovarian cancer," Gynecologic Oncology, vol. 124, no. 3, pp. 582-588, 2012.

[17] H. Iseki, A. Takeda, T. Andoh et al., "Human Arm protein lost in epithelial cancers, on chromosome X 1 (ALEX1) gene is transcriptionally regulated by CREB and $\mathrm{Wnt} / \beta$-catenin signaling," Cancer Science, vol. 101, no. 6, pp. 1361-1366, 2010.

[18] E. Zeller, K. Mock, M. Horn, S. Colnot, M. Schwarz, and A. Braeuning, "Dual-specificity phosphatases are targets of the Wnt $/ \beta$-catenin pathway and candidate mediators of $\beta$ catenin/Ras signaling interactions," Biological Chemistry, vol. 393, no. 10, pp. 1183-1191, 2012.

[19] H. Yao, E. Ashihara, and T. Maekawa, "Targeting the Wnt/ $\beta$ catenin signaling pathway in human cancers," Expert Opinion on Therapeutic Targets, vol. 15, no. 7, pp. 873-887, 2011.

[20] J. R. Jessen, "Noncanonical Wnt signaling in tumor progression and metastasis," Zebrafish, vol. 6, no. 1, pp. 21-28, 2006.

[21] B. T. MacDonald, K. Tamai, and X. He, "Wnt/ $\beta$-Catenin Signaling: Components, Mechanisms, and Diseases," Developmental Cell, vol. 17, no. 1, pp. 9-26, 2009.
[22] M. Hatzfeld, "The armadillo family of structural proteins," International Review of Cytology, vol. 186, no. 1, pp. 179224, 1999.

[23] J. Anaya, "OncoLnc: linking TCGA survival data to mRNAs, miRNAs, and IncRNAs," PeerJ Computer Science, vol. 2, no. 1, article e67, 2016.

[24] L. J. Carithers, K. Ardlie, M. Barcus et al., "A novel approach to high-quality postmortem tissue procurement: The GTEx project," Biopreservation and Biobanking, vol. 13, no. 5, pp. 311319, 2015.

[25] Z. Tang, C. Li, B. Kang, G. Gao, C. Li, and Z. Zhang, "GEPIA: a web server for cancer and normal gene expression profiling and interactive analyses," Nucleic Acids Research, vol. 45, no. W1, pp. W98-W102, 2017.

[26] D. Warde-Farley, S. L. Donaldson, O. Comes et al., “The GeneMANIA prediction server: biological network integration for gene prioritization and predicting gene function," Nucleic Acids Research, vol. 38, suppl_2, pp. W214-W220, 2010.

[27] S. Mostafavi, D. Ray, D. Warde-Farley, C. Grouios, and Q. Morris, "GeneMANIA: a real-time multiple association network integration algorithm for predicting gene function," Genome Biology, vol. 9, Suppl 1, p. S4, 2008.

[28] V. P. Balachandran, M. Gonen, J. J. Smith, and R. P. DeMatteo, "Nomograms in oncology: more than meets the eye," The Lancet Oncology, vol. 16, no. 4, pp. e173-e180, 2015.

[29] A. Subramanian, P. Tamayo, V. K. Mootha et al., "Gene set enrichment analysis: a knowledge-based approach for interpreting genome-wide expression profiles," Proceedings of the National Academy of Sciences of the United States of America, vol. 102, no. 43, pp. 15545-15550, 2005.

[30] V. K. Mootha, C. M. Lindgren, K. F. Eriksson et al., "PGC-1 $\alpha$ responsive genes involved in oxidative phosphorylation are coordinately downregulated in human diabetes," Nature Genetics, vol. 34, no. 3, pp. 267-273, 2003.

[31] A. Liberzon, C. Birger, H. Thorvaldsdóttir, M. Ghandi, J. P. Mesirov, and P. Tamayo, "The Molecular Signatures Database Hallmark Gene Set Collection," Cell Systems, vol. 1, no. 6, pp. 417-425, 2015.

[32] A. Reiner, D. Yekutieli, and Y. Benjamini, "Identifying differentially expressed genes using false discovery rate controlling procedures," Bioinformatics, vol. 19, no. 3, pp. 368-375, 2003.

[33] Y. Benjamini and Y. Hochberg, "Controlling the false discovery rate: a practical and powerful approach to multiple testing," Journal of the Royal Statistical Society: Series B (Methodological), vol. 57, no. 1, pp. 289-300, 1995.

[34] B. Rubinfeld, I. Albert, E. Porfiri, C. Fiol, S. Munemitsu, and P. Polakis, "Binding of GSK3beta to the APC-beta-Catenin complex and regulation of complex assembly," Science, vol. 272, no. 5264, pp. 1023-1026, 1996.

[35] S. R. Krig, V. X. Jin, M. C. Bieda et al., "Identification of genes directly regulated by the oncogene $\mathrm{ZnF} 217$ using chromatin immunoprecipitation (chip)-chip assays," The Journal of Biological Chemistry, vol. 282, no. 13, pp. 97039712, 2007.

[36] C. J. Edwards, J. L. Feldman, J. Beech et al., "Molecular Profile of peripheral blood mononuclear cells from patients with rheumatoid arthritis," Molecular Medicine, vol. 13, no. 1-2, pp. 40-58, 2007.

[37] F. H. Brembeck, M. Rosário, and W. Birchmeier, "Balancing cell adhesion and Wnt signaling, the key role of $\beta$-catenin," 
Current Opinion in Genetics \& Development, vol. 16, no. 1, pp. 51-59, 2006.

[38] T. Reya and H. Clevers, "Wnt signalling in stem cells and cancer," Nature, vol. 434, no. 7035, pp. 843-850, 2005.

[39] M. Katoh, "WNT signaling pathway and stem cell signaling network," Clinical Cancer Research, vol. 13, no. 14, pp. $4042-$ 4045, 2007.

[40] A. E. Bass-Zubek, L. M. Godsel, M. DelSept, and K. J. Green, "Plakophilins: multifunctional scaffolds for adhesion and signaling," Current Opinion in Cell Biology, vol. 21, no. 5, pp. 708-716, 2009.

[41] W. Xu and D. Kimelman, "Mechanistic insights from structural studies of beta-catenin and its binding partners," Journal of Cell Science, vol. 120, no. 19, pp. 3337-3344, 2007.

[42] S. Munemitsu, I. Albert, B. Souza, B. Rubinfeld, and P. Polakis, "Regulation of intracellular beta-catenin levels by the adenomatous polyposis coli (APC) tumor-suppressor protein," Proceedings of the National Academy of Sciences of the United States of America, vol. 92, no. 7, pp. 3046-3050, 1995.

[43] K. H. Goss and J. Groden, "Biology of the adenomatous polyposis coli tumor suppressor," Journal of Clinical Oncology, vol. 18, no. 9, pp. 1967-1979, 2000. 